\title{
ON THE CHEMICAL COMPOSITION OF SEPIOLITE AND PALYGORSKITE
}

\author{
E. García-RøMer ${ }^{1, *}$ AND M. SuÁrez ${ }^{2}$ \\ ${ }^{1}$ Departamento de Cristalografía y Mineralogía, Facultad de Ciencias Geológicas, Universidad Complutense, Madrid, Spain \\ ${ }^{2}$ Departamento de Geología, Universidad de Salamanca, Plaza de la Merced s/n, 37008 Salamanca, Spain
}

\begin{abstract}
Many studies of the chemical composition of sepiolite and palygorskite have been carried out using analytical electron microscopy (AEM). According to the literature, a compositional gap exists between sepiolites and palygorskites, but the results presented here show that they may all be intermediate compositions between two extremes. The results of $>1000 \mathrm{AEM}$ analyses and structural formulae have been obtained for the samples studied ( 22 samples of sepiolite and 21 samples of palygorskite) and indicate that no compositional gap exists between sepiolite and palygorskite. Sepiolite occupies the most magnesic and trioctahedral extreme and palygorskite the most aluminic-magnesic and dioctahedral extreme. Sepiolite and palygorskite with intermediate compositions exist between the two pure extremes: (1) sepiolite with a small proportion of octahedral substitution; (2) palygorskite with a very wide range of substitution (the pure dioctahedral extreme is unusual); and (3) intermediate forms, Al-sepiolite and Mgpalygorskite with similar or the same chemical composition. The chemical compositions of the intermediate forms can be so similar that a certain degree of polymorphism exists between Al-sepiolite and Mg-palygorskite.
\end{abstract}

Key Words-Chemical Composition, Octahedral Sheet, Octahedra cations, Palygorskite, Sepiolite

\section{INTRODUCTION}

The sepielite and palygerskite greup of clay minerals has been studied extensively because they make up a very impertant greup of minerals with a huge number of industrial applications. The number of chemical analyses which have been carried •ut is small, høwever, and many - $f$ the chemical data reperted in the literature are the result of bulk-røck analyses and can be affected by $\bullet$ ther clay minerals and other asseciated minerals as impurities. Published results of micrøanalyses of individual particles $\bullet$ b th sepiølite and palygørskite are quite rare.

According to the literature, a compesitional gap exists between the extremes of these fibrous clay minerals. The triectahedral extreme is sepielite and the more diectahedral extreme, palygerskite (Martín-Vivaldi and Fen๑ll, 1970; Paquet et al., 1987; Galán and Carretere, 1999). The structure of both sepielite and palygorskite contains ribbens of 2:1 phyllesilicates linked by periodic inversion of the apical •xygen of the continu@us tetrahedral sheet every six atems of $\mathrm{Si}$ (three tetrahedral chains) før sepiølite and every føur at॰ms of $\mathrm{Si}$ (tw tetrahedral chains) f $\bullet$ palygerskite. Ribbens (referred to as 'pølisømes' by Krekeler and Guggenheim, 2008) extend parallel to the axis of the fiber. The tetrahedral sheet is continueus acress ribbens but the ectahedral sheet is discontinu $\bullet$ us as a result $\bullet$ the periodic inversion, and terminal •ctahedral cations must complete their coordination sphere with water mølecules referred to as cœerdinated water. Sepiølite has eight possible •ctahedral positions per half unit cell (p.h.u.c.); all are eccupied and its structural formula is $\mathrm{Si}_{12} \bullet_{30} \mathrm{Mg} \mathbf{g}_{8}\left({ }_{\mathrm{H}}\right)_{4}\left(\mathrm{H}_{2}\right)_{2} \cdot 4 \mathrm{H}_{2} \bullet$ (Brauner and Preisinger, 1956). The number of ectahedral positions (p.h.u.c.) in palygorskite is five, $\mathrm{Si}_{8} \bullet_{20} \mathrm{Mg}_{5}\left(\bullet \mathrm{H}_{2}\right)_{2}\left(\mathrm{H}_{2}\right)_{4} \cdot 4 \mathrm{H}_{2} \bullet$ according to the structure prepesed by Bradley (1940), althøugh the five pøsitiens cannet be filled (Serna et al. 1977) and a diectahedral mineral with a structural førmula $\mathrm{Si}_{8} \bullet_{20} \mathrm{Al}_{2} \mathrm{Mg}_{2}(\bullet \mathrm{H})_{2}\left(\mathrm{H}_{2}\right)_{4} \cdot 4 \mathrm{H}_{2} \bullet$ is accepted.

Since the earliest articles published on the chemical compesition of the tw minerals, the pøssibility of a continueus series between sepielite and palygerskite had

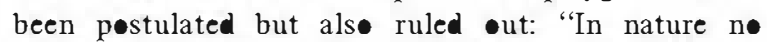
evidence is apparent for a continueus sølid sølution series between the twe, although this may be expected from the postulated similarity of their structures" (Mumpten and R•y, 1958).

Variøus authers have studied the chemical compesitien of sepiolite and palygorskite and they fixed compesitional limits. Martín-Vivaldi and Can๑-Ruíz (1956) suggested that the minerals of the palygorskite sepielite grøup •ccupy the regien of discontinuity between diectahedral and triectahedral minerals. Brauner and Presinger (1956) reperted that the number -f octahedral cations for bulk analyses for sepielite ranges between 6.95 and 8.11 for eight octahedral pesitiens and that ${ }^{\mathrm{VI}} \mathrm{Mg}$ varies between 4.96 and 8.1 .

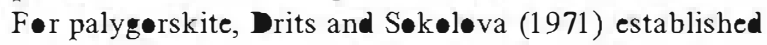
that the sum of octahedral cations for bulk analyses ranges from 3.45 to 4.33 with ${ }^{\mathrm{VI}}(\mathrm{Al}+\mathrm{Fe})$ between 1.12 and 2.3 for five ectahedral pesitions. Paquet et al. (1987) studied 145 individual particles frem palygerskitesmectite and sepiølite-smectite assemblages and affirmed that the octahedral compesition fields of the 
smectites and fibreus clays partly everlap. The sepielite field is clearly in the triectahedral demain, whereas the palygerskite field is both in the diectahedral as well as between the triectahedral and diectahedral demains. Newman and Brown (1987) confirmed that the total number of ectahedral cations of sepielite ranges from 7.01 to 8.01 and between 3.76 and 4.64 for palygerskite, with a mean value of 4.00. Galán and Carreter (1999) published an七ther appreach to cempesitienal limits for sepielite and palygerskite and concluded that sepielite is a true triectahedral mineral, with negligible structural substitutions and eight ectahedral pesitions filled with Mg, while palygerskite is intermediate between di- and triectahedral phylesilicates and its ectahedral sheet contains mainly $\mathrm{Mg}, \mathrm{Al}$, and $\mathrm{Fe}$ (III) with an R2/R3 (where R2 = $\Sigma M(\mathrm{II}), \mathbf{R} 3=\Sigma M(\mathrm{III})$ ) rati clese to 1 and four of the five structural pesitiens eccupied. Miner $\mathrm{Mn}(\mathrm{II}), \mathrm{Fe}(\mathrm{II})$, or $\mathrm{Ni}$ is alse pessible in ectahedral pesitions. If $\mathrm{Ni}>\mathrm{Mg}$, the species is named falcendeite (Springer, 1976; Taulet et al., 2009). Lughlinite is the Na-sepielite, in which $\mathrm{Mg}$ is partially replaced by $\mathrm{Na}$ and alse contains $\mathrm{Na}$ in the channels (Fahey and Axered, 1948). Kadir et al. (2002) found authigenic loughlinite tegether with sepielite in a Neøgene volcan-sedimentary lacustrine envirenment in Mihaliççik-Eskişehir, Turkey. García-Remer et al. (2004) reperted a very Mg-rich palygorskite with 4.36 octahedral cations (p.h.u.c.); Gienis et al. (2006) reperted a very Fe-rich palygerskite; and Garcia-Remer et al. (2007) suggested, from AEM, that Al-rich sepiclite exists in the Alleu-Kagne depesit. In addition, Suarez and García-
R॰mer (2006) reperted that is merphic substitutions in -ctahedral palygerskite sheets eccur •nly in $M 2$ pesitiens. Fe may eccupy $M 2$ pesitiens whereas $\mathrm{Mg}$ can eccupy all possible sites: $M 1, M 2$, and $M 3$. In relation to the chemical compesition of palygerskites, Suarez et al. (2006, 2007) propesed a classification int three types, and their relationship with $\boldsymbol{d}_{200}$ of fering the pessibility of examining the ectahedral compesition of a palygerskite sample using X-ray diffracten (XRD).

Mest of the structural formulae reperted in the literature are summarized here and a large number of analyses of individual particles of beth sepielite and palygerskite from different lecalities has been carried - ut, -btaining representative structural formulae, with the aim of establishing the compesitional limit between the twe minerals.

\section{MATERIALS AND METHODS}

\section{Materials}

The samples studied in the present work (22 samples -f sepielite and 21 samples of palygorskite) came frem different lecalities around the world (Table 1). They show different textural features, with fibers which range fr $\bullet \mathrm{m}<1 \mu \mathrm{m}$ t centimeters in length, and are of different

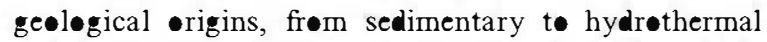
recks. All the samples were of high purity, althøug in søme cases may centain small amøunts of ther minerals as impurities. Samples were either supplied by cemmercial enterprises in the case $\bullet$ re depesits, cellected by

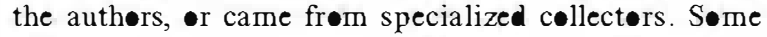

Table 1. Sample sources.

\begin{tabular}{|c|c|c|c|}
\hline Sample & olite - Location & Sample & Location \\
\hline BAT & Batallones (Spain) & BER & Bercimuel (Spain) \\
\hline BOS & Bosnia & BOA & Boavista (Brasil) \\
\hline FIN & Finland & CAS & Cassiar (Canada) \\
\hline GRA & Grant County (USA) & E10 & Esquivias (Spain) \\
\hline HEN & Henan (China) & Ell & Esquivias (Spain) \\
\hline HUN & Hunan (China) & GER & Geraldton (Australia) \\
\hline LIE & Lieyang (China) & LIB & Lisboa (Portugal) \\
\hline MAR & Mara (Spain) & LIL & Lisboa (Portugal) \\
\hline MIER & Eskişehir (Turkey) & MET & Metaline (USA) \\
\hline $\mathrm{MON}$ & Monferrato (Italy) & NIJ & Níjar (Spain) \\
\hline NAM & Namibia & OKE & Okehampton (UK) \\
\hline NEI & Neixiang (China) & PAL & Palygorskaya (Russia) \\
\hline $\mathrm{NEV}$ & Nevada (USA) & PIC & Pics Crossing (Australia) \\
\hline NOR & Norway & SEG & Segovia (Spain) \\
\hline $\mathrm{POL}$ & Polatti (Turkey) & TOR & Torrejón (Spain) \\
\hline ТР० & Polatti (Turkey) & TRA & Los Trancos (Spain) \\
\hline SAN & Santa Cruz (USA) & $\mathrm{Y}$ & Yucatán (México) \\
\hline SOM & Somalia & Y1A & Yucatán (México) \\
\hline VAL & Vallecas (Spain) & Y3 & Yucatán (México) \\
\hline VIC & Vicálvaro (Spain) & Y7 & Yucatán (México) \\
\hline XIX & Xixia (China) & $\mathrm{Y} 8$ & Yucatán (México) \\
\hline YUN & Yunclillos (Spain) & & \\
\hline
\end{tabular}


Table 2. Structural formulae of sepiolites taken from the literature.

\begin{tabular}{|c|c|c|c|c|c|c|c|c|c|c|c|c|c|c|c|c|c|c|c|}
\hline & $\mathrm{Si}$ & ${ }^{\mathrm{IV}} \mathrm{Al}$ & $\mathrm{Fe}^{3+}$ & $\Sigma \tau$ & ${ }^{\mathrm{n}} \mathrm{Al}$ & $\mathrm{Fe}(\mathrm{III})$ & $\mathrm{Fe}(\mathrm{II})$ & $\mathrm{Ti}$ & $\mathrm{Mg}$ & $\mathrm{Cr}$ & $\mathrm{Ni}$ & $\mathrm{Mn}$ & $\mathrm{Cu}$ & $\Sigma_{0}$ & $\mathrm{Ca}$ & $\mathrm{K}$ & $\mathrm{Na}$ & $\mathrm{NH}^{4+}$ & $\mathrm{Cu}$ \\
\hline 1 & 11.81 & •.16 & 0.03 & 12.00 & & 0.47 & 0.13 & & 7.14 & & & & & 7.74 & 0.11 & & & & \\
\hline 2 & 11.61 & 0.22 & •.12 & 11.95 & & & & & 7.35 & & & 0.52 & $\bullet .14$ & 8.01 & & & & & \\
\hline 4 & 11.95 & 0.05 & & 12.00 & 0.01 & 0.07 & & & 7.89 & & & & & 7.97 & 0.20 & & & & \\
\hline 5 & 11.77 & .23 & & 12.00 & 0.02 & 0.25 & •.16 & & 6.98 & & & 0.55 & & 7.96 & & 0.01 & & & \\
\hline 6 & 11.78 & 0.22 & & 12.00 & 0.06 & 0.01 & & & 7.89 & & & & & 7.96 & •.12 & & & & \\
\hline 7 & 11.23 & .24 & 0.53 & 12.00 & & 2.40 & 0.27 & & 4.28 & & & 0.06 & & 7.01 & 0.17 & & & & \\
\hline 9 & 11.54 & 0.46 & & 12.00 & 1.37 & 0.37 & 0.44 & & 4.95 & & & & & 7.13 & & & & & \\
\hline 10 & 11.96 & 0.04 & & 12.00 & 0.17 & 0.46 & 0.26 & & 6.78 & & & & & 7.67 & 0.03 & & 0.03 & & \\
\hline 11 & 11.82 & 0.10 & 0.08 & 12.00 & & & & & 7.73 & & & & & 7.73 & 0.08 & 0.03 & 0.51 & & \\
\hline 12 & 11.93 & 0.07 & & 12.00 & 0.01 & 0.03 & & & 7.96 & & & & & 8.00 & & & 0.03 & & \\
\hline 13 & 11.67 & .22 & 0.11 & 12.00 & & & & & 7.39 & & & 0.53 & & 7.92 & & & & & 0.13 \\
\hline 14 & 11.78 & 0.22 & & 12.00 & 0.05 & $\bullet .01$ & & & 7.90 & & & & & 7.96 & $\bullet .13$ & & & & \\
\hline 15 & 11.87 & & - 13 & 12.00 & & 0.03 & & & 7.90 & & & & & 7.93 & $\bullet .13$ & & & & \\
\hline 16 & 11.80 & 0.16 & 0.04 & 12.00 & & 0.45 & ๑. 14 & & 7.19 & & & & & 7.78 & 0.11 & & & & \\
\hline 19 & 11.70 & 0.26 & 0.04 & 12.00 & 0.06 & & & 0.02 & 7.60 & & & & & 7.68 & & 0.16 & 0.26 & & \\
\hline 20 & 11.64 & 0.36 & & 12.00 & 1.49 & 0.37 & 0.44 & & 4.95 & & & & & 7.25 & & & & 0.45 & \\
\hline 21 & 11.84 & 0.16 & & 12.00 & 0.79 & •.12 & & & 6.04 & & & & & 6.95 & 0.09 & 0.36 & 0.24 & & \\
\hline 22 & 11.96 & 0.04 & & 12.00 & 0.40 & 0.10 & & & 7.04 & & & & & 7.54 & 0.11 & $\bullet .01$ & 0.10 & & \\
\hline 23 & 12.01 & & & 12.01 & 0.05 & 0.05 & & & 7.32 & & & & & 7.42 & 0.46 & & 0.05 & & \\
\hline 24 & 11.80 & 0.11 & 0.09 & 12.00 & & 0.37 & & & 7.51 & & & & & 7.88 & 0.05 & & & & \\
\hline 25 & 11.62 & 0.38 & & 12.00 & 0.04 & 0.06 & & 0.02 & 7.72 & & & & & 7.84 & 0.16 & 0.13 & 0.08 & & \\
\hline 26 & 11.78 & 0.22 & & 12.00 & 0.41 & 0.08 & & & 6.94 & & & & & 7.43 & $\bullet .32$ & 0.13 & 0.11 & & \\
\hline 27 & 11.58 & .42 & & 12.00 & 0.11 & 0.13 & & & 7.53 & & & & & 7.77 & 0.19 & 0.11 & 0.13 & & \\
\hline 28 & 12.02 & & & 12.02 & 0.47 & 0.07 & & 0.02 & 6.89 & & & & & 7.45 & •.11 & 0.16 & •. 12 & & \\
\hline 29 & 11.88 & 0.12 & & 12.00 & 0.29 & 0.08 & & & 7.36 & & & & & 7.73 & 0.12 & & 0.05 & & \\
\hline 30 & 11.75 & 0.21 & & 11.96 & & & & & 8.11 & & & & & 8.11 & & 0.06 & 0.08 & & \\
\hline 31 & 11.28 & .72 & & 12.00 & 0.05 & 0.26 & & & 7.44 & & & & & 7.75 & 0.21 & & 0.51 & & \\
\hline 32 & 11.96 & 0.04 & & 12.00 & 0.20 & 0.10 & & 0.01 & 7.44 & & & & & 7.75 & 0.05 & 0.07 & 0.02 & & \\
\hline 33 & 12.05 & & & 12.05 & 0.05 & 0.01 & & & 7.78 & & & & & 7.84 & 0.03 & & & & \\
\hline 34 & 11.99 & 0.01 & & 12.00 & 0.68 & •.12 & & 0.01 & 6.45 & & & & & 7.26 & 0.09 & 0.25 & 0.22 & & \\
\hline 35 & 11.72 & .28 & & 12.00 & 0.48 & $\bullet .15$ & & 0.04 & 6.50 & & & & & 7.17 & 0.22 & 0.25 & 0.51 & & \\
\hline 39 & 11.83 & 0.08 & 0.08 & 11.99 & & & & & 7.90 & & & & & 7.90 & •.13 & & $\bullet .15$ & & \\
\hline 40 & 11.95 & 0.05 & & 12.00 & & 0.08 & & & 7.69 & & & & & 7.77 & 0.21 & & & & \\
\hline 41 & 11.49 & .33 & 0.18 & 12.00 & & 0.37 & & 0.03 & 7.61 & & & & & 8.01 & & 0.01 & 0.01 & & \\
\hline 42 & 12.00 & & & 12.00 & 0.16 & 0.36 & & 0.02 & 6.99 & 0.07 & 0.04 & & & 7.64 & 0.03 & 0.02 & 0.02 & & \\
\hline
\end{tabular}




\begin{tabular}{|c|c|c|c|c|c|c|c|c|c|c|c|c|c|c|c|c|c|c|c|}
\hline & $\mathrm{Si}$ & ${ }^{\mathrm{rV}} \mathrm{Al}$ & $\mathrm{Fe}^{3+}$ & $\Sigma \tau$ & ${ }^{v_{A l}}$ & $\mathrm{Fe}(\mathrm{III})$ & $\mathrm{Fe}(\mathrm{II})$ & Ti & $\mathrm{Mg}$ & $\mathrm{Cr}$ & $\mathrm{Ni}$ & $\mathrm{Mn}$ & $\mathrm{Cu}$ & $\Sigma o$ & $\mathrm{Ca}$ & $\mathrm{K}$ & $\mathrm{Na}$ & $\mathrm{NH}^{4+}$ & $\mathrm{Cu}$ \\
\hline 43 & 11.99 & 0.01 & & 12.00 & 0.06 & 0.02 & & & 7.84 & & 0.03 & & & 7.95 & & 0.01 & 0.01 & & \\
\hline 44 & 11.80 & .20 & & 12.00 & 0.16 & 1.66 & & & 6.05 & & & & & 7.87 & 0.09 & & 0.07 & & \\
\hline 45 & 12.05 & & & 12.05 & 0.05 & & & & 7.56 & & & & & 7.61 & & 0.02 & 0.24 & & \\
\hline 46 & 11.49 & 0.11 & & 11.60 & & & & & 7.73 & & & & & 7.73 & 1.02 & 0.13 & 0.08 & & \\
\hline 47 & 12.04 & & & 12.04 & & & & & 7.93 & & & & & 7.93 & & & & & \\
\hline 48 & 12.00 & & & 12.00 & & & & & 7.98 & & & & & 7.98 & & & & & \\
\hline 49 & 12.03 & & & 12.03 & & & & & 7.94 & & & & & 7.94 & & & & & \\
\hline 51 & 11.80 & 0.16 & 0.04 & 12.00 & & 0.47 & $\bullet .13$ & & 7.14 & & & & & 7.74 & 0.11 & & & & \\
\hline 52 & 11.54 & 0.46 & & 12.00 & 1.37 & 0.37 & - .44 & & 4.95 & & & & & 7.13 & & & & 0.45 & \\
\hline 53 & 11.96 & 0.04 & & 12.00 & 0.17 & 0.46 & .26 & & 6.78 & & & & & 7.67 & & & 0.03 & & \\
\hline 54 & 11.64 & e.36 & & 12.00 & 0.91 & 0.32 & & & 6.01 & & & & & 7.24 & 0.09 & 0.07 & & & \\
\hline 55 & 11.89 & 0.11 & & 12.00 & 0.38 & 0.19 & & 0.04 & 6.88 & & & & & 7.49 & 0.21 & 0.06 & & & \\
\hline 56 & 11.91 & 0.09 & & 12.00 & 0.73 & 0.23 & & 0.03 & 6.06 & & & & & 7.05 & 0.45 & 0.05 & & & \\
\hline 57 & 11.81 & 0.19 & & 12.00 & 0.67 & 0.25 & & 0.02 & 6.32 & & & & & 7.26 & 0.25 & 0.06 & & & \\
\hline 58 & 11.76 & 0.09 & & 11.85 & & 0.01 & & 0.01 & 8.00 & & & & & 8.02 & & 0.07 & 0.01 & & \\
\hline 59 & 11.42 & 0.46 & & 11.88 & & 0.31 & & 0.05 & 7.67 & & & & & 8.03 & & 0.06 & 0.03 & & \\
\hline 61 & 12.00 & & & 12.00 & & 0.10 & & 0.02 & 7.09 & & & & & 7.21 & & 0.07 & 0.03 & & \\
\hline 62 & 12.00 & & & 12.00 & & 0.08 & & 0.01 & 7.83 & & & & & 7.92 & & 0.05 & 0.02 & & \\
\hline 63 & 12.00 & & & 12.00 & & 0.04 & & & 7.82 & & & & & 7.86 & & 0.07 & 0.01 & & \\
\hline 64 & 12.00 & & & 12.00 & & 0.02 & & & 7.71 & & & & & 7.73 & & 0.06 & 0.01 & & \\
\hline 65 & 11.91 & 0.09 & & 12.00 & & 0.05 & & 0.01 & 7.80 & & & & & 7.86 & 0.05 & 0.06 & 0.04 & & \\
\hline 66 & 11.76 & 0.21 & 0.03 & 12.00 & & 0.05 & & 0.02 & 7.88 & & & & & 7.95 & & 0.13 & 0.09 & & \\
\hline 67 & 12.00 & & & 12.00 & & 0.04 & & 0.01 & 7.60 & & & & & 7.65 & & 0.06 & 0.02 & & \\
\hline 68 & 12.00 & & & 12.00 & 0.02 & 0.15 & & 0.01 & 7.45 & & & 0.05 & & 7.68 & 0.12 & 0.02 & 0.05 & & \\
\hline 69 & 11.98 & 0.02 & & 12.00 & 0.06 & 0.42 & & 0.01 & 6.75 & & & 0.05 & & 7.29 & 0.40 & 0.03 & 0.11 & & \\
\hline 70 & 11.72 & 0.28 & & 12.00 & 0.07 & 0.06 & & $\bullet .01$ & 7.67 & & & 0.01 & & 7.82 & 0.19 & 0.06 & 0.01 & & \\
\hline 71 & 12.00 & & & 12.00 & 0.06 & 0.02 & & 0.01 & 7.39 & & & 0.01 & & 7.49 & 0.07 & 0.02 & 0.04 & & \\
\hline 72 & 11.69 & อ. 31 & & 12.00 & 0.52 & 0.11 & & 0.03 & 6.71 & & & 0.01 & & 7.38 & 0.17 & 0.26 & 0.27 & & \\
\hline 73 & 11.87 & 0.13 & & 12.00 & 0.18 & 1.66 & & 0.01 & 5.22 & & & 0.01 & & 7.08 & 0.08 & 0.01 & 0.01 & & \\
\hline 74 & 12.00 & & & 12.00 & 0.60 & 0.01 & & 0.01 & 6.71 & & & 0.01 & & 7.34 & 0.04 & 0.01 & 0.04 & & \\
\hline 75 & 12.00 & & & 12.00 & 0.39 & 0.09 & & 0.01 & 6.92 & & & 0.01 & & 7.42 & 0.02 & 0.12 & 0.02 & & \\
\hline 76 & 11.80 & 0.20 & & 12.00 & 0.44 & 0.17 & & 0.01 & 6.81 & & & 0.01 & & 7.44 & 0.35 & 0.17 & 0.04 & & \\
\hline 77 & 12.00 & & & 12.00 & 0.08 & 0.02 & & 0.01 & 7.46 & & & 0.01 & & 7.58 & 0.04 & 0.01 & 0.07 & & \\
\hline 81 & 12.01 & & & 12.01 & 0.03 & 1.75 & & 0.01 & 6.05 & & & 0.02 & & 7.86 & 0.06 & 0.02 & 0.08 & & \\
\hline 82 & 11.81 & 0.11 & 0.08 & 12.00 & & 0.37 & & & 7.49 & & & & & 7.86 & 0.05 & & & & \\
\hline 83 & 11.48 & 0.51 & & 11.99 & 0.26 & 1.54 & 0.30 & & 4.45 & & & 0.15 & & 6.70 & 0.19 & 0.69 & 0.77 & & \\
\hline 84 & 11.74 & 0.23 & & 11.97 & 0.16 & 1.65 & & & 6.13 & & & 0.02 & & 7.96 & 0.03 & 0.04 & 0.25 & & \\
\hline 85 & 11.92 & 0.08 & & 12.00 & 0.27 & 0.01 & & & 7.56 & & 0.02 & & & 7.86 & 0.04 & & & & \\
\hline
\end{tabular}


palygerskites were studied previously by the authers, with different aims, and partial results have already been published. In such cases, their structural formulae propesed here have been reviewed with the new data in mind.

Most of the sepiolite and palygorskite formulae from the literature have been collected (Tables 2,3). These analyses shøw great variability having been $\bullet$ btained from different authørs and analyzed by different techniques, including bulk chemical analyses of røcks, which could cøntain admixtures $\bullet$ ther minerals as impurities which are difficult to separate.

\section{Methods}

The high purity of the samples was checked by X-ray diffraction using a Siemens 500 XRD diffractemeter with $\mathrm{CuK} \alpha$ radiation and a graphite menechremater. The samples used were randem-p•wder specimens. P•wders were scanned $\bullet$ ver the range $2-65^{\circ} 20$ at a scan speed $\bullet$ $0.05^{\circ} 20 / 3$ s to determine the mineralegical compesition.

The chemical compesition was $\bullet$ btained by analytical electrøn micrøscopy (AEM) with transmission electron micrescepy (TEM), from samples of great purity. In •rder to ensure the reproducibility of the data, the analyses were

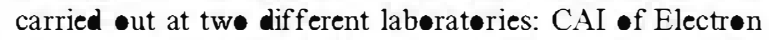
Micrescepy 'Luis Bru' (Cømplutense University, Madrid, Spain) and at the CIC (University of Granada, Spain). At CAI, the data were •btained using a JE $2000 \mathrm{FX}$ micrescepe equipped with a double-tilt sample hølder (up to a maximum of $\pm 45^{\circ}$ ) at an acceleration voltage of $200 \mathrm{kV}$, with $0.5 \mathrm{~mm}$ zeta-axis displacement and $0.31 \mathrm{~nm}$

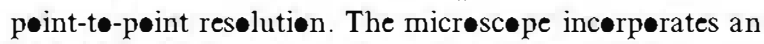
-XF@RD ISIS energy dispersive X-ray spectrømeter $(136 \mathrm{eV}$ resølution at $5.39 \mathrm{keV}$ ) and has its $\bullet$ wn søftware for quantitative analysis. At the CIC (University of Granada), a Philips CM-2 micrøscepe •perated at $200 \mathrm{kV}$ (fitted with an ultrathin windew, solid-state $\mathrm{Si}(\mathrm{Li})$ detecter for energy dispersive X-ray analysis (EAX)) was used. The atemic percentages were calculated by the Cliff-Lorimer thin-film ratiø criteria

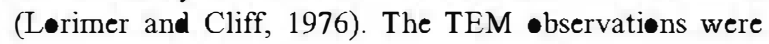
perf $\bullet r m e d$ by depesiting a drøp of diluted suspension $\bullet n$ a micrescepic grid with colledion.

The structural formulae for all analysed particles were calculated $\bullet$ the basis $\bullet \boldsymbol{H}_{20}\left(\bullet \mathrm{H}_{2}\right.$ f $\bullet$ palyg $\boldsymbol{B}_{30}(\bullet \mathrm{H})_{4}$ for sepielites. All the Fe present was cønsidered as Fe(III) (•wing to the limitation of the technique), but the possible existence of Fe(II) cannot be excluded.

\section{RESULTS}

The study of 22 samples of sepielite and 21 of palygerskite by AEM generated $>1000$ analyses. Usually, a small number of the analyses •btained $(\sim 5 \%)$ are errøneus as a consequence of instrumental errørs. Althøugh they are AEM analyses, the influence of søme impurities, specifically silica $\bullet$ cations adsøbed, 
Table 3. Structural formulae of palygorskite taken from the literature.

\begin{tabular}{|c|c|c|c|c|c|c|c|c|c|c|c|c|c|c|c|}
\hline & $\mathrm{Si}$ & ${ }^{\mathrm{rV}} \mathrm{Al}$ & $\Sigma \tau$ & ${ }^{\mathrm{VI}} \mathrm{Al}$ & $\mathrm{Fe}(\mathrm{III})$ & $\mathrm{Fe}(\mathrm{II})$ & $\mathrm{Mg}$ & $\mathrm{Ti}$ & $\mathrm{Fe}(\mathrm{II})$ & $\mathrm{Mn}$ & $\mathrm{Ca}$ & $\Sigma_{0}$ & $\mathrm{Ca}$ & $\mathrm{K}$ & $\mathrm{Na}$ \\
\hline 1 & 7.34 & 0.66 & 8.00 & 2.25 & •.17 & & 1.47 & & & & & 3.89 & 0.21 & & \\
\hline 2 & 7.75 & 0.25 & 8.00 & 2.35 & 0.17 & & 1.29 & & & & & 3.81 & 0.06 & & \\
\hline 3 & 7.61 & 0.39 & 8.00 & 2.26 & 0.23 & & 1.43 & & & & & 3.92 & 0.02 & & \\
\hline 4 & 7.71 & 0.29 & 8.00 & 2.00 & 0.01 & & $1.7 \bullet$ & & & & & 3.71 & & 0.08 & 0.08 \\
\hline 5 & 7.50 & 0.50 & 8.00 & 1.62 & 0.41 & & 1.78 & & & & & 3.81 & 0.34 & & \\
\hline 6 & 8.06 & 0.00 & 8.06 & 2.00 & 0.05 & & 1.62 & & & & & 3.67 & 0.08 & 0.01 & 0.04 \\
\hline 7 & 7.80 & 0.2 & 8.00 & 1.51 & 0.38 & & 2.22 & & & & & 4.11 & & 0.09 & \\
\hline 8 & 7.82 & 0.18 & 8.00 & 1.57 & 0.2 & & 2.04 & & & & & 3.81 & •. 36 & & \\
\hline 9 & 8.09 & 0.00 & 8.09 & 1.57 & 0.00 & & 2.24 & & & & & 3.81 & 0.12 & 0.14 & 0.07 \\
\hline 10 & 7.88 & $\bullet .12$ & 8.00 & 0.95 & 0.42 & & 2.81 & 0.10 & & & & 4.28 & & & \\
\hline 11 & 7.75 & 0.25 & 8.00 & - 12 & 0.10 & 0.47 & 3.84 & 0.08 & & & & 4.61 & $\bullet .17$ & 0.04 & 0.21 \\
\hline 12 & 7.71 & 0.29 & 8.00 & 1.43 & 0.56 & & 2.10 & & & & & 4.09 & & & \\
\hline 13 & 7.86 & 0.14 & 8.00 & 1.84 & 0.40 & & 1.71 & & & & & 3.95 & & & \\
\hline 14 & 8.05 & 0.00 & 8.05 & 1.46 & 0.41 & & 2.09 & & & & & 3.96 & & & \\
\hline 15 & 7.81 & 0.19 & 8.00 & 1.40 & 0.48 & & 1.99 & & & & & 3.87 & 0.04 & 0.06 & 0.32 \\
\hline 16 & 7.80 & 0.2 & 8.00 & 1.13 & 0.87 & & 1.83 & & & & & 3.83 & 0.14 & 0.23 & 0.03 \\
\hline 17 & 7.66 & 0.34 & 8.00 & 1.52 & 0.15 & & 2.65 & & & & & 4.32 & 0.04 & 0.04 & \\
\hline 18 & 8.05 & 0.00 & 8.05 & 1.68 & 0.10 & & 2.2 & & & & & 3.98 & 0.02 & & \\
\hline 19 & 7.85 & 0.15 & 8.00 & 1.15 & 0.38 & & 2.53 & 0.08 & & & & 4.14 & & & \\
\hline 20 & 7.98 & 0.21 & 8.19 & 1.29 & 0.37 & & 1.96 & & 0.03 & & & 3.62 & 0.32 & & \\
\hline 21 & 7.89 & 0.11 & 8.00 & 1.87 & 0.16 & & 1.91 & & & & & 3.94 & 0.05 & & 0.03 \\
\hline 22 & 7.64 & 0.36 & 8.00 & 1.73 & 0.63 & & 1.45 & & & & & 3.81 & 0.08 & & \\
\hline 23 & 7.79 & 0.21 & 8.00 & 1.52 & 0.31 & & 1.89 & 0.05 & & & & 3.77 & •. 31 & 0.05 & 0.08 \\
\hline 24 & 7.43 & 0.57 & 8.00 & 1.58 & 0.65 & & 1.66 & & & & & 3.89 & $\bullet .06$ & - 21 & 0.14 \\
\hline 25 & 7.35 & 0.65 & 8.00 & 1.29 & $\bullet .47$ & & 2.2 & & & & & 3.96 & 0.2 & 0.12 & 0.45 \\
\hline 26 & 7.66 & 0.34 & 8.00 & 1.48 & 0.46 & & 2.02 & 0.03 & & & & 3.99 & 0.05 & 0.15 & - .13 \\
\hline 27 & 7.58 & 0.42 & 8.00 & 0.87 & 0.81 & & 2.42 & 0.08 & & & & 4.18 & 0.08 & 0.08 & 0.03 \\
\hline 28 & 7.50 & $\bullet .50$ & 8.00 & 1.68 & 0.54 & & 1.77 & & & & & 3.99 & & 0.27 & \\
\hline 29 & 7.79 & 0.21 & 8.00 & 1.06 & 0.56 & & 2.46 & 0.02 & & & & 4.10 & $\bullet .06$ & 0.06 & 0.03 \\
\hline 30 & 7.70 & 0.30 & 8.00 & 1.27 & 0.63 & & 2.06 & 0.06 & & & & 4.02 & 0.06 & 0.06 & 0.05 \\
\hline 31 & 7.86 & 0.14 & 8.00 & 2.11 & 0.22 & & 1.12 & & & & & 3.45 & 0.43 & 0.00 & 0.00 \\
\hline 32 & 7.64 & 0.36 & 8.00 & 2.27 & 0.23 & & 1.40 & & & & & 3.90 & 0.02 & 0.00 & 0.00 \\
\hline 33 & 7.61 & 0.39 & 8.00 & 1.81 & 0.00 & & 2.52 & & & & & 4.33 & 0.04 & 0.00 & 0.00 \\
\hline 34 & 7.33 & 0.67 & 8.00 & 2.37 & 0.00 & & 1.69 & & & & & 4.06 & 0.08 & 0.00 & 0.00 \\
\hline 35 & 7.99 & 0.01 & 8.00 & 1.62 & 0.05 & & 1.90 & 0.05 & 0.45 & & & 3.62 & 0.00 & 0.08 & 0.00 \\
\hline 36 & 8.04 & 0.00 & 8.04 & 1.05 & 0.08 & & 2.75 & & 0.00 & & & 3.88 & 0.08 & 0.00 & 0.00 \\
\hline 37 & 7.52 & 0.48 & 8.00 & 2.08 & •. 17 & & 1.37 & 0.04 & 0.00 & & & 3.66 & 0.15 & 0.3 & 0.16 \\
\hline 38 & 7.60 & 0.40 & 8.00 & 1.22 & 0.39 & & 1.75 & & & & & 3.36 & & 0.17 & \\
\hline 39 & 7.78 & 0.22 & 8.00 & 1.57 & 0.47 & & 1.93 & & & & & 3.97 & & 0.22 & \\
\hline 40 & 7.71 & 0.29 & 8.00 & 1.79 & •.62 & & 1.32 & & & & & 3.73 & & 0.46 & \\
\hline 41 & 7.58 & 0.42 & 8.00 & 1.67 & 0.69 & & 1.45 & & & & & 3.81 & & 0.21 & \\
\hline 42 & 7.85 & 0.15 & 8.00 & 1.86 & 0.2 & & 1.81 & & & & & 3.87 & 0.07 & 0.10 & \\
\hline 43 & 7.61 & 0.39 & 8.00 & 0.82 & •.54 & & 2.60 & 0.10 & & & & 4.06 & 0.21 & 0.19 & 0.09 \\
\hline 44 & 7.51 & 0.49 & 8.00 & 1.04 & 0.7 & & 2.43 & & & & & 4.17 & 0.14 & 0.13 & \\
\hline 45 & 7.93 & 0.07 & 8.00 & 1.34 & 0.28 & & 2.48 & & & & & 4.10 & & & \\
\hline 46 & 7.80 & 0.2 & 8.00 & 1.51 & 0.38 & & 2.22 & & & & & 4.11 & & 0.09 & \\
\hline 47 & 7.82 & 0.18 & 8.00 & 1.57 & 0.2 & 0.03 & 2.04 & & & & & 3.84 & •. 36 & & \\
\hline 48 & 7.64 & 0.36 & 8.00 & 1.44 & 0.26 & & 2.46 & & & & & 4.16 & 0.09 & 0.01 & \\
\hline 49 & 7.81 & 0.19 & 8.00 & 1.65 & 0.40 & & 1.88 & 0.02 & & & & 3.95 & 0.04 & 0.13 & \\
\hline 50 & 7.80 & 0.2 & 8.00 & 1.53 & 0.39 & & 1.99 & 0.01 & & & & 3.92 & 0.05 & 0.32 & \\
\hline 51 & 7.62 & 0.38 & 8.00 & 1.58 & 0.39 & & 1.61 & 0.05 & & & & 3.63 & 0.11 & 0.18 & \\
\hline 52 & 7.98 & 0.02 & 8.00 & 1.07 & 0.2 & & 2.83 & 0.06 & & & & 4.16 & 0.10 & 0.08 & \\
\hline 53 & 7.81 & 0.19 & 8.00 & 1.33 & 0.3 & & 2.42 & 0.04 & & & & 4.09 & $\bullet .17$ & 0.08 & \\
\hline 54 & 7.88 & 0.12 & 8.00 & 1.25 & 0.23 & & 2.51 & 0.04 & & & & 4.03 & 0.07 & 0.32 & \\
\hline 55 & 7.87 & 0.13 & 8.00 & 1.46 & 0.40 & & 2.12 & 0.02 & & & & 4.00 & 0.03 & 0.14 & \\
\hline 56 & 7.85 & 0.15 & 8.00 & 1.50 & .23 & & 2.27 & 0.03 & & & & 4.03 & 0.07 & 0.09 & \\
\hline 57 & 7.82 & 0.18 & 8.00 & 1.53 & 0.44 & & 1.98 & 0.01 & & & & 3.96 & 0.01 & 0.03 & \\
\hline 58 & 8.00 & & 8.00 & 1.22 & •. 66 & & 1.75 & 0.09 & & & & 3.72 & & 0.05 & 0.05 \\
\hline 59 & 8.00 & & 8.00 & 1.13 & 0.63 & & 1.98 & 0.08 & & & & 3.82 & 0.03 & 0.06 & 0.05 \\
\hline 60 & 7.20 & 0.80 & 8.00 & 1.36 & 1.31 & & 1.07 & $\bullet .11$ & & 0.01 & & 3.86 & & 0.07 & 0.15 \\
\hline 61 & 7.37 & 0.63 & 8.00 & 1.12 & 1.16 & & 1.43 & 0.09 & & 0.01 & & 3.81 & 0.19 & 0.06 & 0.13 \\
\hline 62 & 8.00 & & 8.00 & 1.29 & 0.38 & & 2.24 & 0.06 & & & & 3.97 & & 0.05 & 0.02 \\
\hline
\end{tabular}




\begin{tabular}{|c|c|c|c|c|c|c|c|c|c|c|c|c|c|c|c|}
\hline & $\mathrm{Si}$ & ${ }^{\mathrm{IV}} \mathrm{Al}$ & $\Sigma \tau$ & ${ }^{\mathrm{VI}} \mathrm{Al}$ & $\mathrm{Fe}(\mathrm{III})$ & $\mathrm{Fe}(\mathrm{II})$ & $\mathrm{Mg}$ & $\mathrm{Ti}$ & $\mathrm{Fe}(\mathrm{II})$ & $\mathrm{Mn}$ & $\mathrm{Ca}$ & $\Sigma_{0}$ & $\mathrm{Ca}$ & $\mathrm{K}$ & $\mathrm{Na}$ \\
\hline 63 & 8.00 & & 8.00 & 1.16 & 0.37 & & 2.43 & 0.06 & & & & 4.02 & 0.08 & 0.05 & 0.03 \\
\hline 64 & 7.65 & 0.35 & 8.00 & 1.48 & 0.51 & & 1.91 & 0.04 & & 0.03 & & 3.97 & 0.09 & 0.11 & \\
\hline 65 & $7.7 \bullet$ & 0.30 & 8.00 & 1.73 & 0.53 & & 1.54 & 0.02 & & $\bullet .03$ & & 3.85 & 0.07 & $\bullet .17$ & \\
\hline 66 & 7.85 & 0.15 & 8.00 & 1.64 & 0.45 & & 1.76 & 0.02 & & 0.01 & & 3.88 & $\bullet .06$ & 0.13 & \\
\hline 67 & 7.64 & 0.36 & 8.00 & 1.35 & 0.58 & & 2.04 & 0.05 & & 0.02 & & 4.04 & 0.08 & 0.11 & \\
\hline 68 & 7.87 & 0.13 & 8.00 & 1.04 & 0.2 & & 3.11 & & & & & 4.35 & 0.02 & 0.03 & 0.08 \\
\hline 69 & 7.85 & 0.14 & 7.99 & 1.47 & 0.25 & & 2.30 & & & & & 4.02 & 0.08 & 0.06 & 0.07 \\
\hline $7 \bullet$ & 7.90 & 0.12 & 8.02 & 1.60 & 0.39 & & 1.98 & & & & & 3.97 & 0.05 & 0.04 & \\
\hline 71 & 7.91 & 0.09 & 8.00 & 1.48 & 0.37 & & 2.25 & & & & & 4.10 & 0.01 & 0.02 & \\
\hline 72 & 7.64 & 0.36 & 8.00 & 2.10 & 0.02 & & 2.14 & & & & & 4.26 & 0.10 & 0.01 & \\
\hline 73 & 7.84 & $\bullet .16$ & 8.00 & 2.00 & 0.06 & & 1.96 & & & 0.04 & & 4.06 & 0.03 & 0.04 & 0.02 \\
\hline 74 & 7.82 & 0.18 & 8.00 & 1.33 & 0.04 & & 3.00 & & & & & 4.37 & & 0.02 & 0.22 \\
\hline 75 & 7.68 & 0.32 & 8.00 & 0.89 & 0.68 & & 2.78 & & & & & 4.35 & 0.86 & 0.04 & 0.10 \\
\hline 76 & 7.94 & 0.06 & 8.00 & 0.68 & 0.07 & & 3.91 & & & & & 4.66 & 0.16 & 0.02 & \\
\hline 77 & 7.71 & 0.2 & 8.00 & 1.47 & 0.06 & & 2.74 & & & & & 4.27 & 0.17 & & 0.37 \\
\hline 78 & 7.96 & 0.04 & 8.00 & 1.71 & 0.07 & & 2.33 & & & & & 4.11 & 1.45 & 0.05 & \\
\hline 79 & 7.77 & 0.23 & 8.00 & 1.50 & 0.33 & & 1.90 & & & & & 3.73 & 0.47 & & 0.19 \\
\hline 80 & 8.02 & & 8.02 & 0.92 & 0.49 & & $2.7 \bullet$ & 0.06 & & & & 4.17 & 0.03 & & \\
\hline 81 & 8.13 & & 8.13 & 1.84 & 0.15 & & 1.69 & & & & & 3.68 & 0.04 & 0.01 & \\
\hline 82 & 8.02 & & 8.02 & 0.92 & 0.49 & & $2.7 \bullet$ & 0.06 & & & & 4.17 & 0.03 & & \\
\hline 83 & 8.13 & & 8.13 & 1.84 & 0.15 & & 1.69 & & & & & 3.68 & 0.04 & 0.01 & \\
\hline 84 & 7.81 & 0.19 & 8.00 & 1.66 & 0.36 & & 1.83 & & & & & 3.85 & 0.01 & 0.45 & \\
\hline 85 & 7.60 & 0.40 & 8.00 & 1.58 & 0.40 & & 1.79 & & & & & 3.77 & 0.24 & 0.40 & \\
\hline 86 & 7.85 & 0.15 & 8.00 & 1.72 & 0.07 & & 2.10 & 0.03 & & & & 3.92 & 0.23 & & \\
\hline 87 & 7.83 & 0.17 & 8.00 & 1.58 & 0.19 & 0.03 & 2.04 & & & & & 3.84 & 0.36 & & \\
\hline 88 & 7.82 & 0.18 & 8.00 & 2.02 & 0.09 & & 1.97 & & & & & 4.08 & 0.01 & 0.01 & \\
\hline 89 & 7.80 & 0.2 & 8.00 & 1.92 & 0.11 & & 2.09 & & & & & 4.12 & 0.01 & & 0.01 \\
\hline 90 & 7.83 & 0.17 & 8.00 & 1.86 & 0.18 & & 1.61 & & & & & 3.65 & & 0.03 & 0.12 \\
\hline 91 & 7.49 & 0.50 & 7.99 & 1.19 & 0.26 & & 2.66 & & & & & 4.11 & 0.28 & 0.02 & 0.03 \\
\hline 92 & 8.00 & & 8.00 & 0.82 & 0.48 & & 2.70 & 0.06 & & & & 4.06 & & & \\
\hline 93 & 8.00 & & 8.00 & 1.68 & 0.23 & & 2.12 & & & & & 4.03 & & & \\
\hline 94 & 8.01 & & 8.01 & 1.61 & 0.53 & & 2.35 & & & & & 4.49 & 0.11 & 0.28 & \\
\hline 95 & 7.95 & 0.05 & 8.00 & 1.49 & 0.61 & & 1.73 & & & & & 3.83 & & & \\
\hline 96 & 7.64 & 0.36 & 8.00 & 0.70 & 1.05 & & 2.23 & 0.02 & & & & 4.00 & 0.18 & 0.06 & 0.03 \\
\hline 97 & 8.00 & & 8.00 & 1.89 & 0.05 & & 1.99 & 0.01 & & & & 3.94 & 0.07 & & \\
\hline 98 & 7.79 & 0.21 & 8.00 & 1.64 & 0.42 & & 1.90 & & & & & 3.96 & 0.03 & 0.12 & \\
\hline 99 & 7.27 & 0.73 & 8.00 & 0.94 & 0.02 & & 1.90 & & & & 0.01 & 2.87 & & & \\
\hline 100 & 7.27 & $\bullet .73$ & 8.00 & 0.94 & 0.02 & & 1.90 & & & & 0.01 & 2.87 & & & \\
\hline 101 & 7.48 & 0.52 & 8.00 & 1.24 & 0.94 & & 1.77 & 0.03 & & & & 3.98 & & & \\
\hline 102 & 7.64 & 0.36 & 8.00 & 1.44 & 0.26 & & 2.46 & & & & & 4.16 & & & \\
\hline 103 & 7.80 & 0.2 & 8.00 & 1.13 & 0.87 & & 1.83 & & & & & 3.83 & $\bullet .14$ & 0.23 & 0.03 \\
\hline 104 & 8.00 & & 8.00 & 1.19 & 0.33 & & 2.60 & & & & & 4.12 & 0.06 & 0.10 & 0.02 \\
\hline 105 & 7.95 & 0.05 & 8.00 & 1.63 & 0.25 & & 2.25 & & & & & 4.13 & & 0.06 & 0.01 \\
\hline 106 & 7.08 & 0.98 & 8.06 & 2.64 & 0.29 & 1.60 & 2.10 & & & & & 6.63 & 0.10 & & \\
\hline 107 & 8.02 & & 8.02 & 1.91 & $\bullet .04$ & & 2.01 & & & & & 3.96 & 0.01 & & 0.07 \\
\hline 108 & 7.85 & 0.15 & 8.00 & 1.57 & 0.24 & & 2.21 & & & & & 4.02 & 0.03 & 0.11 & \\
\hline 109 & 7.95 & 0.05 & 8.00 & 1.93 & 0.08 & & 1.92 & & & & & 3.93 & 0.03 & 0.01 & 0.09 \\
\hline 110 & 7.74 & 0.26 & 8.00 & 0.81 & 1.12 & & 2.11 & & & & & 4.04 & 0.01 & 0.06 & 0.15 \\
\hline 111 & 7.80 & 0.2 & 8.00 & 2.00 & 0.80 & & 1.10 & & & & & 3.90 & & 0.40 & \\
\hline 112 & 7.43 & 0.58 & 8.01 & 1.49 & 0.83 & & 1.54 & & & & & 3.86 & & & \\
\hline 113 & 7.38 & 0.62 & 8.00 & 0.96 & 0.62 & & 2.86 & & & & & 4.44 & & & \\
\hline
\end{tabular}

1-11 compiled by Newman and Brown (1987); 12-14 from Galán and Carretero (1999); $15-45$ compiled by Galán and Carretero (1999); 46-48 compiled by Jones and Galán (1991); 49-57 from Zaaboub et al. (2005); 58-63 from Torres-Ruiz et al. (1994); 64-67 from Jamoussi et al. (2003); 68-71 from García-Romero et al. (2004); $72-79$ from Post and Crawford (2007); 80-81 from Neaman and Singer (2000); 82-83 from Weaver and Polland (1973); 84-85 from Weaver (1984); 86-87 from Imai and Otsuka (1984); 88-89 from Post and Heaney (2008); 90-91 from Corma et al. (1987); 92 from Singer and Norrish (1974); 93 from Giiven (1992); 94 from Verrecchia and Le Coustumer (1996); 95 from López-Galindo (1987); 96 from Akbulut and Kadir (2003); 97 from Tien (1973); 98 from López-Galindo et al. (1996); 99 from Artioli et al. (1994); 100 from Artioli and Gali (1994); 101 from Li et al. (2007); 102 Galán et al. (1975); 103 from Siddiki (1984); 104 from Chahi et al. (2002); 105 from Giusteto et al. (2006); 106 from Suárez et al. (1994); 107 from Suárez and García-Romero (2006); 108 from Suárez et al. (2007); 109 from García-Romero et al. (2006); 110 from Gionis et al. (2006); 111 from Magalhaes et al. (2008); 112 from López-Galindo et al. (2008); and 113 from Chen et al. (2008). 
cannet be ruled out completely. In the case when impurities were present, accurate comparisen of chemical compesitiens was precluded. Prier to btaining the mean structural formulae, a certain number of the analyses obtained was eliminated, using the following criteria. First, the formulae that not have a goed balance of charges were deleted, assuming instrumental errers in such cases. Alse remeved were all these

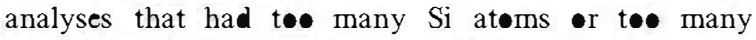
-ctahedral cations. Whether a formula for sepiøle with $>12$ at $\bullet$ of $\mathrm{Si} \bullet \mathrm{f} \bullet \mathrm{r}$ palygerskite with $>8$ at $\bullet$ ms $\mathrm{Si}$ is the result of an instrumental errer •r because of adserbed amerpheus silica is unknøwn. In any case, excess silica which is to great cannet cerrespend to tetrahedral pesitions. Taking int accunt that sepiolite has 12 tetrahedral pesitions (p.h.u.c.) the formulae with $\geqslant 12.2 \mathrm{Si}$ atems were remeved. Samples may centain a

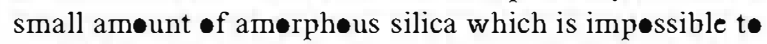
avıid. Equally, sepiølite has eight ectahedral pesitions and the analyses with $>8$ ectahedral cations were eliminated. For the same reasen, for palygerskites, analyses containing $\geqslant 8.2$ Si $\bullet \geqslant 5 \bullet$ ctahedral pøsitions -ccupied (p.h.u.c.) were remeved. Remaining were 1223 definitive analytical data for the structural formulae calculatiens (454 from sepielite and 779 frem palygerskite). Appreximately half of the data remeved corre-

Table 4. Chemical composition (mean values, wt.\% oxides) of the sepiolite samples studied.

\begin{tabular}{|c|c|c|c|c|c|c|c|c|c|c|}
\hline & & $\mathrm{SiO}_{2}$ & $\mathrm{Al}_{2} \mathrm{O}_{3}$ & $\mathrm{Fe}_{2} \mathrm{O}_{3}$ & $\mathrm{Mg} \bullet$ & $\mathrm{TiO}_{2}$ & $\mathrm{Ca}$ & $\mathrm{Na}$ & $\mathrm{K}_{2}$ & $\Sigma$ \\
\hline \multirow[t]{2}{*}{ BAT } & $N-19$ & 67.48 & 4.31 & 2.13 & 23.41 & 1.12 & 0.94 & $\bullet .12$ & $\bullet .56$ & 100.07 \\
\hline & STDV & 1.73 & 1.29 & 1.19 & 2.70 & 1.18 & 0.91 & 0.33 & 0.56 & 0.32 \\
\hline \multirow[t]{2}{*}{ BOS } & $N-14$ & 66.70 & 2.75 & 2.14 & 25.33 & & 1.54 & & 1.34 & 99.80 \\
\hline & STDV & 3.47 & 1.80 & 1.59 & 2.88 & & 1.44 & & 1.61 & 0.83 \\
\hline \multirow[t]{2}{*}{ FIN } & $N-22$ & 66.46 & 2.82 & 3.22 & 25.60 & 0.04 & 1.08 & 0.17 & .63 & 100.01 \\
\hline & STDV & 2.05 & 1.21 & 1.18 & 1.70 & 0.10 & 0.97 & .26 & 0.84 & $\bullet .13$ \\
\hline \multirow[t]{2}{*}{ GRA } & $N-13$ & 69.01 & 0.49 & 0.44 & 29.19 & & 0.34 & .43 & 0.10 & 100.00 \\
\hline & STDV & 0.68 & 0.34 & 0.44 & 0.84 & & 0.33 & 0.44 & 0.08 & 0.01 \\
\hline \multirow[t]{2}{*}{$\mathrm{HEN}$} & $N-33$ & 68.54 & 0.83 & 0.59 & 29.27 & 0.01 & 0.31 & .21 & 0.19 & 99.95 \\
\hline & STDV & 0.75 & 0.57 & 0.48 & 0.95 & 0.06 & 0.36 & .43 & 0.27 & 0.10 \\
\hline \multirow[t]{2}{*}{ HUN } & $N-24$ & 66.09 & 5.24 & 2.61 & 23.13 & & 0.78 & 0.94 & 1.19 & 99.97 \\
\hline & STDV & 1.97 & 2.21 & 1.08 & 2.04 & & 0.92 & 0.83 & 0.83 & 0.06 \\
\hline \multirow[t]{2}{*}{ LIE } & $N-15$ & 69.43 & 5.97 & 2.44 & 21.38 & 0.01 & 0.31 & 0.39 & 0.04 & 99.89 \\
\hline & STDV & 0.86 & 2.14 & 1.29 & 2.84 & 0.03 & 0.12 & 0.12 & 0.09 & 0.17 \\
\hline \multirow[t]{2}{*}{ MAR } & $N-30$ & 67.57 & 3.40 & 2.04 & 26.22 & 0.13 & 0.24 & 0.04 & 0.27 & 100.03 \\
\hline & STDV & 1.80 & 1.87 & 1.45 & 1.55 & 0.32 & 0.3 & 0.08 & 0.31 & 0.24 \\
\hline \multirow{2}{*}{ MER } & $N-6$ & 69.04 & 0.25 & 0.07 & 30.57 & & 0.02 & & 0.06 & 100.00 \\
\hline & STDV & 0.44 & 0.27 & 0.11 & 0.33 & & 0.04 & & 0.09 & 0.01 \\
\hline \multirow[t]{2}{*}{$\mathrm{MON}$} & $N-23$ & 68.59 & 1.43 & 1.61 & 27.72 & & 0.34 & & 0.18 & 99.95 \\
\hline & STDV & 1.37 & 1.17 & 0.81 & 1.14 & & 0.18 & & 0.2 & 0.19 \\
\hline \multirow[t]{2}{*}{ NAM } & $N-20$ & 68.74 & 1.18 & 0.70 & 28.78 & & 0.55 & & 0.16 & 100.11 \\
\hline & STDV & 1.28 & 0.70 & 0.92 & 1.12 & & 0.50 & & 0.27 & 0.3 \\
\hline \multirow[t]{2}{*}{ NEI } & $N-25$ & 66.77 & 2.20 & 1.88 & 27.48 & & 0.87 & 0.01 & 0.76 & 99.96 \\
\hline & STDV & 3.85 & 2.07 & 2.45 & 2.55 & & 1.25 & 0.06 & 0.93 & 0.09 \\
\hline \multirow[t]{2}{*}{$\mathrm{NEV}$} & $N-31$ & 66.52 & 2.35 & 1.53 & 28.01 & & 0.88 & 0.11 & 0.59 & 99.99 \\
\hline & STDV & 2.72 & 1.08 & 1.07 & 1.97 & & 1.2 & 0.25 & 0.41 & 0.07 \\
\hline \multirow[t]{2}{*}{ NOR } & $N-26$ & 68.49 & 1.12 & 0.38 & 28.78 & 0.15 & 0.34 & 0.19 & 0.46 & 99.91 \\
\hline & STDV & 1.33 & 1.38 & 0.45 & 1.52 & 0.30 & 0.54 & .35 & 1.09 & 0.27 \\
\hline \multirow[t]{2}{*}{$\mathrm{POL}$} & $N-22$ & 65.39 & 8.35 & 4.56 & 18.58 & 1.02 & 1.18 & & 0.78 & 99.88 \\
\hline & STDV & 0.40 & 2.09 & 2.34 & 4.06 & 0.88 & 0.53 & & 0.40 & 0.18 \\
\hline \multirow[t]{2}{*}{ SAN } & $N-12$ & 69.02 & 0.20 & 0.44 & 29.64 & & 0.3 & 0.27 & 0.13 & 99.99 \\
\hline & STDV & 0.56 & 0.2 & 0.29 & 0.75 & & 0.21 & .41 & 0.18 & 0.03 \\
\hline \multirow[t]{2}{*}{ SOM } & $N-22$ & 68.38 & 1.14 & 0.53 & 29.30 & & 0.27 & 0.12 & 0.24 & 100.00 \\
\hline & STDV & 1.28 & 0.74 & 0.59 & 0.75 & & 0.38 & 0.2 & 0.38 & 0.07 \\
\hline \multirow[t]{2}{*}{ TPO } & $N-11$ & 69.02 & 0.34 & 0.35 & 29.95 & & 0.21 & 0.13 & & 100.00 \\
\hline & SATDV & 0.57 & 0.36 & 0.31 & 0.61 & & 0.2 & 0.2 & & 0.01 \\
\hline \multirow[t]{2}{*}{ VAL } & $N-17$ & 68.01 & 2.01 & 0.64 & 28.20 & 0.31 & 0.29 & 0.12 & 0.34 & 99.80 \\
\hline & STDV & 2.22 & 1.11 & 0.83 & 1.41 & 0.52 & 0.56 & 0.46 & 0.57 & 0.54 \\
\hline \multirow[t]{2}{*}{ VIC } & $N-2$ & 67.25 & 2.37 & 1.79 & 27.72 & 0.02 & 0.50 & 0.04 & 0.27 & 99.97 \\
\hline & STDV & 2.30 & 1.29 & 2.16 & 1.96 & 0.09 & 0.67 & 0.17 & .4 & 0.19 \\
\hline \multirow[t]{2}{*}{ XIX } & $N-6$ & 69.67 & 0.05 & 0.89 & 29.08 & & 0.11 & 0.2 & & 100.00 \\
\hline & STDV & 0.40 & 0.12 & 0.44 & 0.58 & & 0.17 & 0.49 & & 0.01 \\
\hline YUN & $N-2$ & 69.50 & 1.04 & 0.30 & 28.78 & 0.00 & 0.15 & 0.10 & 0.05 & 100.00 \\
\hline & STDV & 0.44 & 0.23 & 0.15 & 0.36 & 0.02 & 0.07 & 0.14 & 0.07 & 0.01 \\
\hline
\end{tabular}


spended to analyses with toe much silica, probably adserbed amerphøus silica but pessibly als related to instrumental errers.

The chemical compesitions of the samples can be compared frem mean values of exide percentages (Tables 4, 5). Sepielite has a mean $\mathrm{SiO}_{2}$ centent which ranges between $65.39 \%$ (POL and FIN samples) and $69.67 \%$ (XIX sample). The Mgo content varies greatly between $30.57 \%$ in the mest magnesic sample (MER) and $18.58 \%$ in the POL sample which, in accerd with the $\mathrm{Mg}$ content, has the greatest propertion of $\mathrm{Al}_{2} \mathrm{O}_{3}$ $(8.35 \%)$. Three samples have an $\mathrm{Al}_{2} \mathbf{O}_{3}$ centent of $<4 \%$. $\mathrm{Fe}_{2} \mathrm{O}_{3}$ ranges between $3.22 \%$ and $\mathbf{0 . 0 7 \%}$ (FIN and MER, respectively), although $70 \%$ of the samples have $<2 \%$ of this exide. The contents of ether exides such as $\mathrm{Ti}_{2}, \mathrm{~K}_{2}$, and $\mathrm{Na}_{2} \bullet$ are generally small $\bullet$ zer. The mean contents are somewhat greater $(\sim 1 \%$ $\mathrm{TiO}_{2}$ in BAT, BOS, and POL, - $1.94 \% \mathrm{Na}_{2}$ in HUN) but the standard deviation is similar the mean value which indicates the variability of these exides in the AEM analyses. $\mathrm{CaO}$ is present in all the sepielites studied with variable content and a large standard deviation

Palygerskite samples have $\mathrm{SiO}_{2}$ centents which range between $72.93 \%$ (LIL sample) and 68.90\% (BOA sample) and shew a great variability in terms of the

Table 5. Chemical composition (mean values, wt.\% oxides) of the palygorskite samples studied.

\begin{tabular}{|c|c|c|c|c|c|c|c|c|c|c|}
\hline & & $\mathrm{SiO}_{2}$ & $\mathrm{Al}_{2} \mathrm{O}_{3}$ & $\mathrm{Fe}_{2} \mathrm{O}_{3}$ & Mge & $\mathrm{TiO}_{2}$ & $\mathrm{Ca}$ & $\mathrm{Na}$ & $\mathrm{K}_{2} \bullet$ & $\Sigma$ \\
\hline \multirow[t]{2}{*}{ ATT } & $N-8$ & 69.58 & 12.11 & 3.27 & 13.73 & 0.00 & 0.65 & 0.26 & $\bullet .36$ & 99.95 \\
\hline & STDV & 0.76 & 0.98 & 0.53 & 0.99 & 0.01 & 0.30 & 0.37 & 0.24 & 0.07 \\
\hline \multirow[t]{2}{*}{ BER } & $N-6$ & 70.04 & 12.97 & 4.54 & 11.76 & & 0.43 & & 0.25 & 100.00 \\
\hline & STDV & 1.08 & 2.03 & 0.47 & 1.50 & & 0.27 & & 0.25 & 0.01 \\
\hline \multirow[t]{2}{*}{ BOA } & $N-72$ & 68.90 & 13.88 & 4.16 & 11.28 & & 0.36 & 0.95 & 0.64 & 100.15 \\
\hline & STDV & 2.21 & 1.44 & 1.38 & 1.13 & & - .51 & 0.84 & 0.66 & 0.31 \\
\hline \multirow[t]{2}{*}{ CAS } & $N-39$ & 71.38 & 14.46 & 1.76 & 10.75 & & 0.48 & - .62 & 0.54 & 100.02 \\
\hline & STDV & 1.68 & 0.95 & 0.93 & 0.75 & & $\bullet .35$ & 0.79 & 0.54 & 0.47 \\
\hline \multirow[t]{2}{*}{ E 10} & $N-41$ & 70.42 & 7.52 & 1.64 & 19.82 & & 0.25 & & 0.28 & 99.92 \\
\hline & STDV & 1.46 & 1.57 & 1.08 & 1.89 & & 0.50 & & 0.37 & 0.40 \\
\hline \multirow[t]{2}{*}{ E 11} & $N-96$ & 69.44 & 10.04 & 2.05 & 17.33 & $\bullet .01$ & 0.38 & 0.47 & 0.31 & 100.03 \\
\hline & STDV & 7.21 & 6.45 & 0.92 & 2.50 & 0.03 & 0.55 & 1.37 & 0.33 & 0.59 \\
\hline \multirow{2}{*}{ LIB } & $N-12$ & 71.88 & 12.31 & 1.74 & 13.37 & 0.33 & 0.11 & 0.21 & 0.02 & 99.99 \\
\hline & STDV & 0.92 & 0.70 & 0.58 & 0.64 & 0.56 & 0.10 & 0.20 & 0.04 & 0.03 \\
\hline \multirow[t]{2}{*}{ LIL } & $N-2$ & 72.93 & 14.56 & 0.29 & 11.75 & 0.01 & $\bullet .17$ & 0.36 & 0.02 & 100.10 \\
\hline & STDV & 0.93 & 0.68 & 0.26 & 0.95 & 0.03 & 0.2 & - 64 & 0.03 & 0.36 \\
\hline \multirow[t]{2}{*}{ MET } & $N-12$ & 72.16 & 13.51 & 0.57 & 13.04 & & - 64 & & 0.10 & 100.00 \\
\hline & STDV & 0.86 & 0.95 & 0.81 & 1.05 & & 0.61 & & 0.24 & 0.01 \\
\hline \multirow[t]{2}{*}{ NIJ } & $N-13$ & 71.71 & 12.61 & 2.12 & 12.57 & $\bullet .06$ & 0.09 & 0.85 & 0.03 & 100.05 \\
\hline & STDV & 1.00 & 3.42 & 1.65 & 1.67 & 0.19 & 0.06 & 0.43 & 0.04 & 0.18 \\
\hline \multirow[t]{2}{*}{ OKE } & $N-48$ & 71.26 & 15.88 & 0.38 & 11.38 & 0.01 & 0.15 & 0.63 & 0.11 & 99.80 \\
\hline & STDV & 1.23 & 0.65 & 0.43 & 1.01 & 0.02 & 0.26 & 0.56 & 0.26 & 0.42 \\
\hline \multirow[t]{2}{*}{ PAL } & $N-63$ & 71.41 & 15.03 & 0.22 & 12.37 & 0.00 & 0.37 & 0.45 & 0.15 & 100.00 \\
\hline & STDV & 1.71 & 1.37 & 0.34 & 1.70 & 0.02 & 0.43 & 0.54 & 0.31 & 0.01 \\
\hline \multirow[t]{2}{*}{ PIC } & $N-52$ & 70.08 & 9.99 & 5.54 & 13.21 & 0.14 & 0.18 & 0.74 & 0.07 & 99.96 \\
\hline & STDV & 0.88 & 0.71 & 0.51 & 0.66 & 0.33 & 0.19 & 0.49 & 0.11 & 0.15 \\
\hline \multirow[t]{2}{*}{ SEG } & $N-46$ & 71.07 & 15.84 & 0.68 & 11.16 & 0.01 & - 41 & 0.74 & 0.08 & 99.99 \\
\hline & STDV & 1.56 & 1.06 & 0.51 & 0.96 & 0.05 & 0.65 & 0.65 & 0.14 & 0.25 \\
\hline \multirow[t]{2}{*}{ TOR } & $N-17$ & 70.08 & 11.59 & 4.48 & 13.60 & & 0.06 & & 0.08 & 99.90 \\
\hline & STDV & 0.61 & 0.83 & 0.59 & 0.92 & & 0.11 & & 0.14 & 0.13 \\
\hline \multirow[t]{2}{*}{ TRA } & $N-2$ & 71.42 & 9.21 & 1.83 & 16.91 & 0.09 & 0.38 & & 0.13 & 99.97 \\
\hline & STDV & 1.00 & 0.72 & 0.36 & 1.05 & 0.52 & 0.40 & & 0.17 & 0.13 \\
\hline \multirow[t]{2}{*}{$\mathrm{Y} \bullet$} & $N-28$ & 70.25 & 12.17 & 4.01 & 12.49 & 0.06 & 0.19 & $\bullet .37$ & 0.43 & 99.97 \\
\hline & STDV & 1.90 & 1.82 & 1.81 & 1.25 & 0.12 & 0.2 & 0.65 & - 62 & 0.07 \\
\hline \multirow[t]{2}{*}{ Y1A } & $N-30$ & 71.49 & 11.83 & 1.96 & 14.30 & & - 26 & 0.09 & 0.08 & 100.01 \\
\hline & STDV & 1.01 & 1.78 & 0.89 & 1.63 & & 0.37 & 0.29 & 0.17 & 0.03 \\
\hline \multirow[t]{2}{*}{ Y3 } & $N-14$ & 71.72 & 11.92 & 1.60 & 14.08 & 0.07 & 0.12 & 0.18 & 0.31 & 10.00 \\
\hline & STDV & 1.03 & 0.95 & 0.43 & 0.96 & 0.10 & 0.15 & 0.27 & 0.3 & 0.01 \\
\hline \multirow[t]{2}{*}{ Y7 } & $N-15$ & 71.26 & 12.75 & 2.08 & 13.27 & 0.05 & 0.21 & 0.31 & 0.08 & 98.16 \\
\hline & STDV & 1.41 & 1.04 & 0.96 & 0.87 & 0.18 & - 31 & 0.53 & 0.18 & 4.45 \\
\hline \multirow[t]{2}{*}{ Y8 } & $N-15$ & 70.60 & 13.46 & 2.43 & 12.91 & 0.07 & 0.06 & 0.05 & 0.42 & 10.00 \\
\hline & STDV & 1.06 & 1.23 & 0.43 & 0.78 & 0.14 & 0.09 & 0.09 & 0.28 & 0.01 \\
\hline YUC & $N-11$ & 71.45 & 11.36 & 3.02 & 14.01 & & 0.07 & 0.06 & 0.03 & 100.00 \\
\hline & STDV & 1.47 & 1.16 & 1.52 & 1.17 & & 0.24 & 0.19 & 0.08 & 0.01 \\
\hline
\end{tabular}


contents of the exides of the main octahedral cations. $\mathrm{Al}_{2} \mathrm{O}_{3}$ ranges between 15.88 and $7.52 \%$ ( KE and $\mathrm{E} 1$, respectively) and $\mathrm{Mg}$ between 19.82 and $10.75 \%$ (E1 and CAS, respectively). All of the samples studied contain $\mathrm{Fe}_{2} \mathrm{O}_{3}$ with the amount reaching $5.54 \%$ in the PIC sample, with standard deviation equal to 151 , which indicates that in general all analyses correspending to this sample contain a large amount of $\mathrm{Fe}$. $\mathrm{TiO}_{2}$ is absent from nine samples and in the rest the content is very small. $\mathrm{Na}_{2} \bullet, \mathrm{K}_{2} \bullet$, and $\mathrm{Ca}$ appear as miner compnents, $\mathrm{Ca}$ occurring in all samples studied. Both minerals present a great variability in terms of amounts -f the main oxides (Tables 4, 5). In fact, if the amounts - $f$ xides of the main octahedral cations are plotted (Figure 1), the analyses appear to be projected in a continueus region witheut separation between the twe minerals.

The mean values of each cation, the total, tetrahedral, and octahedral contents (p.h.u.c), and the standard deviation for all are shown together with $\mathrm{Si} / \mathrm{Mg}$ and R3/R2 relations (Tables 6,7 ). The number of $\mathrm{Si}$ atoms ranges from 11.5 to 12.11 for sepiolite and from 7.88 to 8.06 for palygorskite. The number of total octahedral cations ranges from 6.87 to 7.95 for sepiolite and from 3.35 to 4.4 for palygorskite. These values correspond to - $6-14 \%$ ctahedral vacancies for sepiolite and between 12 and $23.6 \%$ for palygorskite. Clearly, $\mathrm{Mg}$ is the main octahedral cation in sepiolite (4.88-7.92) and this mineral contains only miner amounts of $\mathrm{Al}$ (0.1-1.24) and Fe(IM) (1-.43). Palygerskite shows greater octahedral variability: $\mathrm{M}$ (1.79-3.34), $\mathrm{Al}(\bullet 2-1.99)$, and $\mathrm{Fe}(\mathrm{min})(.2-47)$. Mest of the analyses of each sample range between narrow limits, as indicated by the small values for standard deviation

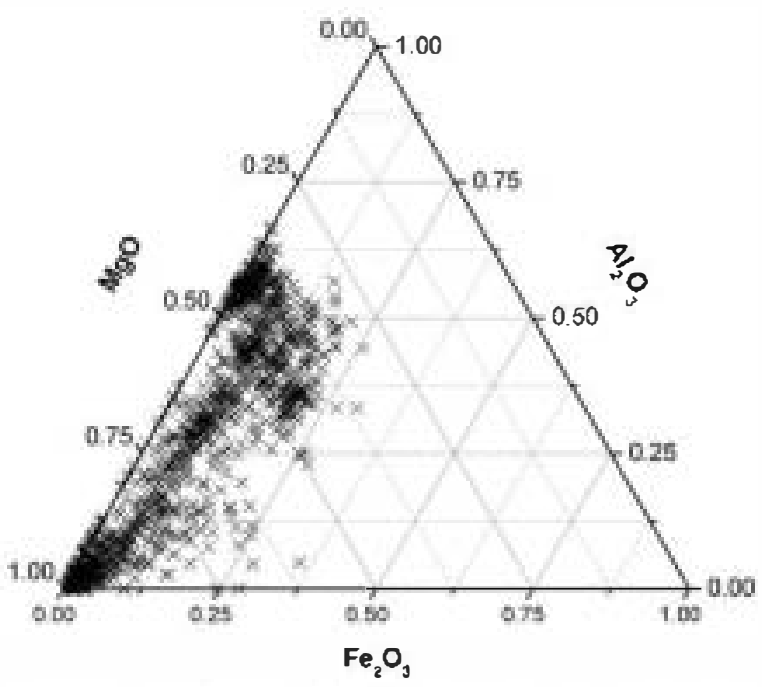

Figure 1. AFM diagram for the sepiolites and palyzorskites studied. All points analyzed are projected between the two extremes, but with ne separation between ata corresponding to sepiolites and palyorskites.
(Tables 6,7). Very small amounts of Ti appear in the mean structural formulae of several sepiolites. $\mathrm{Ca}$ is always present as an exchangeable cation and $\mathrm{Na}$ and $\mathrm{K}$ are present in mest samples.

\section{DISCUSSION}

Due to the large number of samples studied in the present work, several chemical variations were found. The structural formulae propesed for the samples studied (Tables 8, 9) are from the mean values of each cation -btained (Tables 6, 7). Regarding the tetrahedral compesition, theoretical sepiolite has twelve tetrahedral positions and palygorskite eight, although a few of these positions can be partially filled by $\mathrm{Al}$ and sepiolite could have $F e$ in tetrahedral positions. A certain number of samples of sepiolite have $\mathrm{Fe}(\mathrm{II})$ as a tewahedral cation (Table 6), in very small amounts $(\mathbf{0 4 0 4 )}$ and with standard deviation even greater than the mean. Regarding the ectahedral content, the natural palygerskites show a wider range of substitutions than sepiolites, which can correspond to different types. The MER, TPO, and SAN sepiolites have a structural formula close to the theoretical, both for the tetrahedral and the octahedral content. The MER sample is the mest 'perfect' sepiolite frem these data, in goed agreement with these published by Ece (1998) for sepilite from the same locality. Twelve samples contain variable amounts of terahedral $\mathrm{Al}$, with $>7$ atems of $\mathrm{M}$ p.h.u.c. (between 90 and $95 \%$ -f the octahedral pesitions occupied by $\mathrm{Mg}$ ) and miner amounts of $\mathrm{Al}$ and $\mathrm{Fe}(\mathrm{IM})$; the remainder of the samples (MAR, BOS, FIN, BAT, HUN, and POL) are characterized by the greater octahedral substitution and these six samples als present tetrahedral substitution. These mest Al-rich and/or Fe-rich sepilites have between 61 and $85 \%$ of the ectahedral positions occupied by $\mathrm{Mg}$

When comparing these results with those which appear in the literature (Table 2), some sepiolites show the same characteristics: smaller $\mathrm{Mg}$ content than the ideal composition, due to substitution by $\mathrm{Al}$ and/or Fe(II). This seems to indicate that Al-rich sepiolite is not sø rare. The Mg content vs. total octahedral content was plotted (Figure 2), both for data from the literature and for those from the present study. The same distribution for the two types of data was found. As expected, most of the points plotted in the region with the greatest $\mathrm{Mg}$ values with the largest number of -ctahedral cations because sepiolite may not have -ctahedral substitution (Galán and Carretere, 1999). A certain number of analyses correspond to the mest dioctahedral extremes and the smallest $\mathrm{M}$ contents and equate to Al-rich sepiolites such as that referred to by Regers et al. (1956). Imai and Otsuka (1084) reperted Fe-rich palygorskite, and Garcia-Remer et al. (2007) reperted very $\mathrm{Al}$-rich sepielite together with a very $\mathrm{M}$ rich palygerskite in the Allou Kagne depesit (Senegal). Looking at the data both from the literature and from 
Table 6. Mean values of tetrahedral and octahedral cations, and $\mathrm{Si} / \mathrm{Mg}$ and $\mathbf{R} 3 / \mathbf{R} 2$ relations for the sepiolite samples, calculated from the structural formulae.

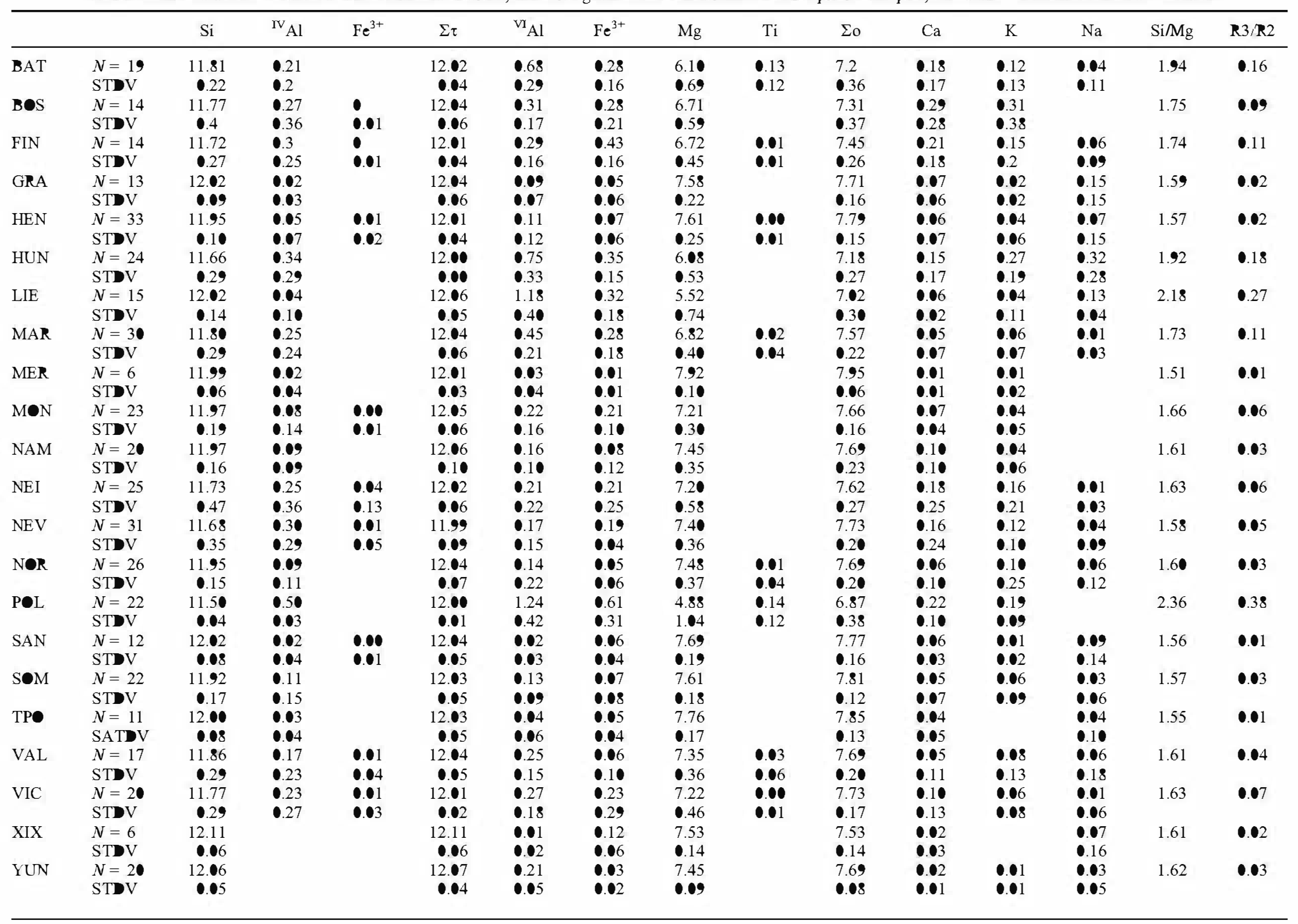


AEM in this study, sepielite can be classified int twe groups: sepielite and Al-sepielite. A limit for these tw groups can be established from the ectahedral eccupancy and Al-sepielites are these that have $>10 \% \bullet$ oftahedral pesitions vacant and $>.5^{\mathrm{VI}} \mathrm{Al}$ atems (Table 6).

Palygerskite has greater cempesitional variation than sepielite. The ideal palygerskite has filled four of its five ectahedral pesitions (2 $\mathrm{Mg}$ and $2 \mathrm{Al}$ ). Accerding to Suárez et al. (2007), based on the ectahedral compesitien, palygerskite could be of three different types: (1) Ideal palygerskite, with an ectahedral cempesition near to the ideal palygerskite, similar centents of $\mathrm{Al}$ and $\mathrm{Mg}$, and negligible substitutiens. (2) Cemmen palygerskite, where the ${ }^{\mathrm{VI}} \mathrm{Al}$ content is less than in the ideal formula and as a consequence the $\mathrm{Mg}$ content is greater, but the number of octahedral cations is clese to 4 (vacant ectahedral pesitions = 1). Althøugh Al may be partially substituted by $\mathrm{Fe}$ (III) and/•r Mg, this type of palygerskite has diectahedral character. (3) Magnesic palygerskite is the mest triectahedral extreme. The number of -ctahedral cations is $>4$ (vacant ectahedral pesitions 1 ). Palygerskite can, en ecasion, be Fe-rich, such as the

Table 7. Mean values of te crahedral and octahedral cations, Si/Mg and R3/R2 relations for the samples of palygorskite calculated from the structural formulae.

\begin{tabular}{|c|c|c|c|c|c|c|c|c|c|c|c|c|c|c|}
\hline & & $\mathrm{Si}$ & ${ }^{\mathrm{rV}} \mathrm{Al}$ & $\Sigma \tau$ & ${ }^{\mathrm{VI}} \mathrm{Al}$ & $\mathrm{Fe}^{3+}$ & $\mathrm{Mg}$ & $\mathrm{Ti}$ & $\Sigma o$ & $\mathrm{Ca}$ & $\mathrm{K}$ & $\mathrm{Na}$ & $\mathrm{Si} / \mathrm{Mg}$ & $\mathbf{R} 3 / \mathbf{R} 2$ \\
\hline ATT & $\begin{array}{l}N-8 \\
\text { STDV }\end{array}$ & $\begin{array}{l}7.86 \\
0.07\end{array}$ & $\begin{array}{l}0.14 \\
0.07\end{array}$ & $\begin{array}{l}8.00 \\
0.01\end{array}$ & $\begin{array}{l}1.47 \\
0.08\end{array}$ & $\begin{array}{l}0.28 \\
0.04\end{array}$ & $\begin{array}{l}2.31 \\
0.16\end{array}$ & $\begin{array}{l}0.00 \\
0.01\end{array}$ & $\begin{array}{l}4.06 \\
0.07\end{array}$ & $\begin{array}{l}0.08 \\
0.04\end{array}$ & $\begin{array}{l}0.05 \\
0.03\end{array}$ & $\begin{array}{l}0.06 \\
0.08\end{array}$ & 3.40 & 0.76 \\
\hline BER & $\begin{array}{l}N-6 \\
\text { STDV }\end{array}$ & $\begin{array}{l}7.90 \\
0.12\end{array}$ & $\begin{array}{l}0.12 \\
0.09\end{array}$ & $\begin{array}{l}8.02 \\
0.04\end{array}$ & $\begin{array}{l}1.60 \\
0.20\end{array}$ & $\begin{array}{l}0.39 \\
0.04\end{array}$ & $\begin{array}{l}1.98 \\
0.25\end{array}$ & & $\begin{array}{l}3.97 \\
0.11\end{array}$ & $\begin{array}{l}0.05 \\
0.03\end{array}$ & $\begin{array}{l}0.04 \\
0.04\end{array}$ & & 3.99 & $1 . \bullet 1$ \\
\hline BOA & $\begin{array}{l}N-72 \\
\text { STDV }\end{array}$ & $\begin{array}{l}7.80 \\
0.17\end{array}$ & $\begin{array}{l}0.21 \\
0.17\end{array}$ & $\begin{array}{l}8.00 \\
0.02\end{array}$ & $\begin{array}{l}1.64 \\
\bullet .17\end{array}$ & $\begin{array}{l}\bullet .35 \\
\bullet .12\end{array}$ & $\begin{array}{l}1.91 \\
0.19\end{array}$ & & $\begin{array}{l}3.91 \\
0.11\end{array}$ & $\begin{array}{l}\bullet .04 \\
\bullet .06\end{array}$ & $\begin{array}{l}0.09 \\
0.10\end{array}$ & $\begin{array}{l}0.21 \\
0.19\end{array}$ & 4.08 & 1.04 \\
\hline CAS & $\begin{array}{l}N-39 \\
\text { STDV }\end{array}$ & $\begin{array}{l}7.98 \\
0.13\end{array}$ & $\begin{array}{l}0.06 \\
0.09\end{array}$ & $\begin{array}{l}8.04 \\
0.06\end{array}$ & $\begin{array}{l}1.88 \\
0.11\end{array}$ & $\begin{array}{l}\bullet .15 \\
\bullet .08\end{array}$ & $\begin{array}{l}1.79 \\
0.12\end{array}$ & & $\begin{array}{l}3.81 \\
0.11\end{array}$ & $\begin{array}{l}\bullet .06 \\
\bullet .04\end{array}$ & $\begin{array}{l}0.08 \\
0.08\end{array}$ & $\begin{array}{l}0.12 \\
0.18\end{array}$ & 4.46 & 1.13 \\
\hline E 10 & $\begin{array}{l}N-41 \\
\text { STDV }\end{array}$ & $\begin{array}{l}7.95 \\
\bullet .13\end{array}$ & $\begin{array}{l}0.08 \\
0.10\end{array}$ & $\begin{array}{l}8.03 \\
0.05\end{array}$ & $\begin{array}{l}0.92 \\
0.19\end{array}$ & $\begin{array}{l}0.14 \\
0.09\end{array}$ & $\begin{array}{l}3.34 \\
\bullet .32\end{array}$ & & $\begin{array}{l}4.40 \\
0.16\end{array}$ & $\begin{array}{l}0.03 \\
0.06\end{array}$ & $\begin{array}{l}0.04 \\
0.05\end{array}$ & & 2.38 & - 32 \\
\hline E 11 & $\begin{array}{l}N-96 \\
\text { STDV }\end{array}$ & $\begin{array}{l}7.92 \\
0.15\end{array}$ & $\begin{array}{l}0.10 \\
0.13\end{array}$ & $\begin{array}{l}8.02 \\
0.04\end{array}$ & $\begin{array}{l}1.14 \\
0.20\end{array}$ & $\begin{array}{l}0.18 \\
0.08\end{array}$ & $\begin{array}{l}2.92 \\
0.41\end{array}$ & $\begin{array}{l}0.00 \\
0.01\end{array}$ & $\begin{array}{l}4.25 \\
\text { 0.21 }\end{array}$ & $\begin{array}{l}0.04 \\
0.05\end{array}$ & $\begin{array}{l}0.04 \\
0.05\end{array}$ & $\begin{array}{l}0.06 \\
0.11\end{array}$ & 2.71 & 0.45 \\
\hline LIB & $\begin{array}{l}N-12 \\
\text { STDV }\end{array}$ & $\begin{array}{l}7.98 \\
0.09\end{array}$ & $\begin{array}{l}0.03 \\
0.05\end{array}$ & $\begin{array}{l}8.01 \\
0.06\end{array}$ & $\begin{array}{l}1.61 \\
\bullet .07\end{array}$ & $\begin{array}{l}0.16 \\
\bullet .02\end{array}$ & $\begin{array}{l}2.27 \\
\bullet .11\end{array}$ & $\begin{array}{l}0.01 \\
0.01\end{array}$ & $\begin{array}{l}4.05 \\
1.57\end{array}$ & $\begin{array}{l}0.02 \\
0.01\end{array}$ & & $\begin{array}{l}0.05 \\
0.04\end{array}$ & 3.53 & 0.78 \\
\hline LIL & $\begin{array}{l}N-20 \\
\text { STDV }\end{array}$ & $\begin{array}{l}8.06 \\
0.07\end{array}$ & $\begin{array}{l}0.03 \\
0.13\end{array}$ & $\begin{array}{l}8.10 \\
0.14\end{array}$ & $\begin{array}{l}1.89 \\
0.08\end{array}$ & $\begin{array}{l}0.02 \\
0.02\end{array}$ & $\begin{array}{l}1.95 \\
0.17\end{array}$ & & $\begin{array}{l}3.85 \\
0.12\end{array}$ & $\begin{array}{l}0.01 \\
0.02\end{array}$ & & $\begin{array}{l}0.07 \\
0.13\end{array}$ & 4.13 & 0.98 \\
\hline MET & $\begin{array}{l}N-12 \\
\text { STDV }\end{array}$ & $\begin{array}{l}8.02 \\
0.08\end{array}$ & $\begin{array}{l}0.02 \\
0.04\end{array}$ & $\begin{array}{l}8.04 \\
0.05\end{array}$ & $\begin{array}{l}1.75 \\
\bullet .11\end{array}$ & $\begin{array}{l}0.05 \\
0.07\end{array}$ & $\begin{array}{l}2.16 \\
\bullet .17\end{array}$ & & $\begin{array}{l}3.95 \\
0.13\end{array}$ & $\begin{array}{l}0.08 \\
0.07\end{array}$ & $\begin{array}{l}0.01 \\
0.02\end{array}$ & & 3.71 & 0.83 \\
\hline NIJ & $\begin{array}{l}N-13 \\
\text { STDV }\end{array}$ & $\begin{array}{l}8.02 \\
0.11\end{array}$ & $\begin{array}{l}0.04 \\
0.06\end{array}$ & $\begin{array}{l}8.06 \\
0.07\end{array}$ & $\begin{array}{l}1.62 \\
\bullet .40\end{array}$ & $\begin{array}{l}0.18 \\
0.14\end{array}$ & $\begin{array}{l}2.10 \\
0.30\end{array}$ & & $\begin{array}{l}3.90 \\
0.13\end{array}$ & $\begin{array}{l}\bullet .01 \\
\bullet .01\end{array}$ & $\begin{array}{l}0.00 \\
0.01\end{array}$ & $\begin{array}{l}0.19 \\
0.10\end{array}$ & 3.82 & 0.86 \\
\hline OKE & $\begin{array}{l}N-48 \\
\text { STDV }\end{array}$ & $\begin{array}{l}7.92 \\
0.10\end{array}$ & $\begin{array}{l}0.09 \\
0.08\end{array}$ & $\begin{array}{l}8.01 \\
0.02\end{array}$ & $\begin{array}{l}1.99 \\
0.09\end{array}$ & $\begin{array}{l}\bullet .03 \\
0.04\end{array}$ & $\begin{array}{l}1.88 \\
0.17\end{array}$ & & $\begin{array}{l}3.91 \\
0.11\end{array}$ & $\begin{array}{l}0.02 \\
0.03\end{array}$ & $\begin{array}{l}0.02 \\
0.04\end{array}$ & $\begin{array}{l}0.14 \\
0.12\end{array}$ & 4.21 & 1.07 \\
\hline PAL & $\begin{array}{l}N-63 \\
\text { STDV }\end{array}$ & $\begin{array}{l}7.93 \\
\bullet .15\end{array}$ & $\begin{array}{l}0.08 \\
0.13\end{array}$ & $\begin{array}{l}8.02 \\
0.05\end{array}$ & $\begin{array}{l}1.89 \\
0.20\end{array}$ & $\begin{array}{l}0.02 \\
0.03\end{array}$ & $\begin{array}{l}2.05 \\
0.30\end{array}$ & 0.00 & $\begin{array}{l}3.95 \\
0.16\end{array}$ & $\begin{array}{l}0.04 \\
0.05\end{array}$ & $\begin{array}{l}0.02 \\
0.04\end{array}$ & $\begin{array}{l}0.10 \\
0.12\end{array}$ & 3.87 & 0.93 \\
\hline PIC & $\begin{array}{l}N-52 \\
\text { STDV }\end{array}$ & $\begin{array}{l}7.96 \\
0.08\end{array}$ & $\begin{array}{l}0.05 \\
0.06\end{array}$ & $\begin{array}{l}8.01 \\
0.03\end{array}$ & $\begin{array}{l}1.28 \\
0.08\end{array}$ & $\begin{array}{l}\bullet .47 \\
\bullet .04\end{array}$ & $\begin{array}{l}2.24 \\
\bullet .11\end{array}$ & $\begin{array}{l}0.01 \\
0.03\end{array}$ & $\begin{array}{l}4.01 \\
0.07\end{array}$ & $\begin{array}{l}0.02 \\
0.02\end{array}$ & $\begin{array}{l}0.01 \\
0.02\end{array}$ & $\begin{array}{l}0.16 \\
0.11\end{array}$ & 3.55 & 0.78 \\
\hline SEG & $\begin{array}{l}N-46 \\
\text { STDV }\end{array}$ & $\begin{array}{l}7.90 \\
0.14\end{array}$ & $\begin{array}{l}0.11 \\
\bullet .12\end{array}$ & $\begin{array}{l}8.02 \\
0.04\end{array}$ & $\begin{array}{l}1.97 \\
0.11\end{array}$ & $\begin{array}{l}\bullet .06 \\
\bullet .04\end{array}$ & $\begin{array}{l}1.85 \\
0.17\end{array}$ & & $\begin{array}{l}3.88 \\
0.11\end{array}$ & $\begin{array}{l}0.05 \\
0.08\end{array}$ & $\begin{array}{l}0.01 \\
0.02\end{array}$ & $\begin{array}{l}\bullet .16 \\
\bullet .14\end{array}$ & 4.27 & 1.10 \\
\hline TOR & $\begin{array}{l}N-17 \\
\text { STDV }\end{array}$ & $\begin{array}{l}7.88 \\
0.08\end{array}$ & $\begin{array}{l}0.12 \\
0.07\end{array}$ & $\begin{array}{l}8.00 \\
0.01\end{array}$ & $\begin{array}{l}1.43 \\
0.10\end{array}$ & $\begin{array}{l}0.38 \\
0.05\end{array}$ & $\begin{array}{l}2.28 \\
0.16\end{array}$ & & $\begin{array}{l}4.09 \\
0.06\end{array}$ & $\begin{array}{l}0.01 \\
\bullet .01\end{array}$ & $\begin{array}{l}0.02 \\
0.03\end{array}$ & & 3.46 & 0.79 \\
\hline TRA & $\begin{array}{l}N-20 \\
\text { STDV }\end{array}$ & $\begin{array}{l}8.02 \\
0.08\end{array}$ & $\begin{array}{l}0.02 \\
0.08\end{array}$ & $\begin{array}{l}8.03 \\
0.05\end{array}$ & $\begin{array}{l}1.20 \\
0.11\end{array}$ & $\begin{array}{l}0.15 \\
0.03\end{array}$ & $\begin{array}{l}2.83 \\
0.18\end{array}$ & $\begin{array}{l}0.01 \\
0.05\end{array}$ & $\begin{array}{l}4.19 \\
0.12\end{array}$ & $\begin{array}{l}0.05 \\
0.05\end{array}$ & $\begin{array}{l}0.02 \\
0.03\end{array}$ & & 2.83 & 0.48 \\
\hline Y० & $\begin{array}{l}N-28 \\
\text { STDV }\end{array}$ & $\begin{array}{l}7.93 \\
0.17\end{array}$ & $\begin{array}{l}0.11 \\
0.13\end{array}$ & $\begin{array}{l}8.04 \\
0.05\end{array}$ & $\begin{array}{l}1.51 \\
0.18\end{array}$ & $\begin{array}{l}0.34 \\
0.16\end{array}$ & $\begin{array}{l}2.10 \\
0.21\end{array}$ & $\begin{array}{l}0.01 \\
0.01\end{array}$ & $\begin{array}{l}3.96 \\
0.15\end{array}$ & $\begin{array}{l}0.02 \\
0.02\end{array}$ & $\begin{array}{l}0.06 \\
0.09\end{array}$ & $\begin{array}{l}0.08 \\
0.14\end{array}$ & 3.78 & 0.88 \\
\hline Y $1 \mathrm{~A}$ & $\begin{array}{l}N-30 \\
\text { STDV }\end{array}$ & $\begin{array}{l}7.99 \\
0.09\end{array}$ & $\begin{array}{l}0.04 \\
0.06\end{array}$ & $\begin{array}{l}8.03 \\
0.05\end{array}$ & $\begin{array}{l}1.52 \\
\bullet .20\end{array}$ & $\begin{array}{l}\bullet .16 \\
\bullet .07\end{array}$ & $\begin{array}{l}2.38 \\
0.28\end{array}$ & & $\begin{array}{l}4.07 \\
0.11\end{array}$ & $\begin{array}{l}\bullet .03 \\
\bullet .04\end{array}$ & $\begin{array}{l}0.01 \\
0.02\end{array}$ & $\begin{array}{l}0.02 \\
0.06\end{array}$ & 3.36 & 0.71 \\
\hline Y3 & $\begin{array}{l}N-14 \\
\text { STDV }\end{array}$ & $\begin{array}{l}8.02 \\
0.09\end{array}$ & $\begin{array}{l}0.03 \\
0.04\end{array}$ & $\begin{array}{l}8.04 \\
0.06\end{array}$ & $\begin{array}{l}1.54 \\
0.11\end{array}$ & $\begin{array}{l}0.13 \\
0.04\end{array}$ & $\begin{array}{l}2.35 \\
\bullet .16\end{array}$ & $\begin{array}{l}\bullet .01 \\
0.01\end{array}$ & $\begin{array}{l}4.03 \\
0.10\end{array}$ & $\begin{array}{l}0.01 \\
0.02\end{array}$ & $\begin{array}{l}0.04 \\
0.04\end{array}$ & $\begin{array}{l}0.04 \\
0.06\end{array}$ & 3.41 & 0.71 \\
\hline Y7 & $\begin{array}{l}N-15 \\
\text { STDV }\end{array}$ & $\begin{array}{l}7.96 \\
\bullet .12\end{array}$ & $\begin{array}{l}0.07 \\
0.09\end{array}$ & $\begin{array}{l}8.03 \\
0.05\end{array}$ & $\begin{array}{l}1.62 \\
\bullet .13\end{array}$ & $\begin{array}{l}0.17 \\
\bullet .09\end{array}$ & $\begin{array}{l}2.21 \\
\bullet .15\end{array}$ & $\begin{array}{l}0.00 \\
0.02\end{array}$ & $\begin{array}{l}4.01 \\
0.14\end{array}$ & $\begin{array}{l}\bullet .03 \\
0.04\end{array}$ & $\begin{array}{l}0.01 \\
0.03\end{array}$ & $\begin{array}{l}0.07 \\
0.11\end{array}$ & 3.60 & 0.81 \\
\hline Y8 & $\begin{array}{l}N-15 \\
\text { STDV }\end{array}$ & $\begin{array}{l}7.91 \\
0.11\end{array}$ & $\begin{array}{l}0.09 \\
0.10\end{array}$ & $\begin{array}{l}8.00 \\
0.02\end{array}$ & $\begin{array}{l}1.68 \\
0.08\end{array}$ & $\begin{array}{l}0.20 \\
0.04\end{array}$ & $\begin{array}{l}2.16 \\
\bullet .13\end{array}$ & $\begin{array}{l}0.01 \\
0.01\end{array}$ & $\begin{array}{l}4.05 \\
0.07\end{array}$ & $\begin{array}{l}\bullet .01 \\
\bullet .01\end{array}$ & $\begin{array}{l}0.07 \\
0.04\end{array}$ & $\begin{array}{l}0.01 \\
0.02\end{array}$ & 3.66 & 0.87 \\
\hline YUC & $\begin{array}{l}N-11 \\
\text { STDV }\end{array}$ & $\begin{array}{l}8.00 \\
0.11\end{array}$ & $\begin{array}{l}0.03 \\
0.08\end{array}$ & $\begin{array}{l}8.04 \\
0.05\end{array}$ & $\begin{array}{l}1.47 \\
\bullet .17\end{array}$ & $\begin{array}{l}\bullet .23 \\
\bullet .15\end{array}$ & $\begin{array}{l}2.34 \\
\text { 0.21 }\end{array}$ & & $\begin{array}{l}4.04 \\
0.13\end{array}$ & $\begin{array}{l}0.04 \\
0.10\end{array}$ & $\begin{array}{l}0.00 \\
0.01\end{array}$ & $\begin{array}{l}\bullet .01 \\
0.04\end{array}$ & 3.42 & •.73 \\
\hline
\end{tabular}


Table 8. Structural formulae proposed for the sepiolites studied.

\begin{tabular}{|c|c|c|c|c|c|c|c|c|c|c|c|}
\hline & $\mathrm{Si}$ & ${ }^{\mathrm{rV}} \mathrm{Al}$ & $\Sigma \tau$ & ${ }^{\mathrm{v}} \mathrm{Al}$ & $\mathrm{Fe}^{3+}$ & $\mathrm{Mg}$ & $\mathrm{Ti}$ & $\Sigma_{0}$ & $\mathrm{Ca}$ & $\mathrm{K}$ & $\mathrm{Na}$ \\
\hline BAT & 11.81 & 0.19 & 12.00 & 0.68 & 0.28 & 6.10 & 0.13 & 7.19 & 0.18 & 0.12 & 0.04 \\
\hline BOS & 11.77 & 0.23 & 12.00 & 0.31 & 0.28 & 6.71 & & 7.30 & 0.29 & 0.31 & \\
\hline FIN & 11.72 & 0.28 & 12.00 & •. 12 & $\bullet .61$ & 6.66 & & 7.46 & 0.25 & 0.17 & 0.05 \\
\hline GRA & 12.00 & & $12 . \bullet 0$ & 0.09 & 0.05 & 7.58 & & 7.72 & 0.07 & 0.02 & 0.15 \\
\hline $\mathrm{HEN}$ & 11.95 & 0.05 & 12.00 & 0.11 & 0.07 & 7.61 & & 7.79 & 0.06 & 0.04 & 0.07 \\
\hline HUN & 11.66 & 0.34 & 12.00 & 0.75 & 0.35 & 6.08 & & 7.18 & 0.15 & 0.27 & 0.32 \\
\hline LIE & 12.00 & & 12.00 & 1.18 & 0.32 & 5.52 & & 7.02 & 0.06 & 0.04 & 0.13 \\
\hline MAR & 11.80 & 0.2 & 12.00 & 0.45 & 0.28 & 6.82 & 0.02 & 7.57 & 0.05 & 0.06 & 0.01 \\
\hline MER & 11.99 & 0.01 & 12.00 & 0.03 & 0.01 & 7.92 & & 7.96 & 0.01 & 0.01 & \\
\hline $\mathrm{MON}$ & 11.97 & 0.03 & 12.00 & 0.22 & .21 & 7.21 & & 7.64 & 0.07 & 0.04 & \\
\hline NAM & 11.97 & 0.03 & 12.00 & 0.16 & 0.08 & 7.45 & & 7.69 & 0.10 & 0.04 & \\
\hline NEI & 11.73 & 0.22 & 11.95 & 0.22 & .21 & 7.20 & & 7.62 & 0.18 & 0.16 & 0.01 \\
\hline $\mathrm{NEV}$ & 11.68 & 0.32 & 12.00 & 0.17 & 0.19 & 7.40 & & 7.76 & 0.16 & 0.12 & 0.04 \\
\hline NOR & 11.95 & 0.05 & 12.00 & 0.14 & 0.05 & 7.48 & 0.01 & 7.68 & 0.06 & 0.10 & 0.06 \\
\hline POL & 11.50 & 0.50 & 12.00 & 1.24 & 61 & 4.88 & 0.14 & 6.87 & 0.22 & 0.19 & \\
\hline SAN & 12.00 & & 12.00 & 0.02 & 0.06 & 7.69 & & 7.77 & $\bullet .06$ & 0.01 & 0.09 \\
\hline SOM & 11.92 & 0.08 & 12.00 & 0.13 & 0.07 & 7.61 & & 7.81 & 0.05 & 0.06 & 0.03 \\
\hline ТРО & 12.00 & & 12.00 & 0.04 & 0.05 & 7.76 & & 7.85 & 0.04 & & 0.04 \\
\hline VAL & 11.84 & $\bullet .16$ & 12.00 & 0.25 & 0.06 & 7.35 & 0.03 & 7.69 & 0.05 & 0.08 & 0.06 \\
\hline VIC & 11.77 & .23 & 12.00 & 0.27 & .23 & 7.22 & & 7.72 & 0.10 & 0.06 & 0.1 \\
\hline XIX & 12.00 & & 12.00 & 0.10 & $\bullet .12$ & 7.53 & & 7.66 & 0.02 & & 0.07 \\
\hline YUN & 12.00 & & 12.00 & 0.27 & 0.03 & 7.45 & & 7.69 & 0.02 & 0.01 & 0.03 \\
\hline MAX & 12.00 & 0.50 & & 1.24 & •.61 & 7.92 & 0.14 & 7.96 & 0.29 & 0.31 & 0.32 \\
\hline MIN & 11.50 & 0.00 & & 0.01 & 0.00 & 4.88 & 0.00 & 6.87 & 0.01 & 0.00 & 0.00 \\
\hline MEAN & 11.87 & 0.13 & & 0.32 & 0.19 & 7.06 & 0.02 & 7.57 & 0.10 & 0.09 & 0.06 \\
\hline
\end{tabular}

Table 9. Structural formulae proposed for the palygorskites studied.

\begin{tabular}{|c|c|c|c|c|c|c|c|c|c|c|c|}
\hline & $\mathrm{Si}$ & ${ }^{\mathrm{rV}} \mathrm{Al}$ & $\Sigma \tau$ & ${ }^{\mathrm{VI}} \mathrm{Al}$ & $\mathrm{Fe}^{3+}$ & $\mathrm{Mg}$ & $\mathrm{Ti}$ & $\Sigma o$ & $\mathrm{Ca}$ & $\mathrm{K}$ & $\mathrm{Na}$ \\
\hline ATT & 7.86 & 0.14 & 8.00 & 1.47 & 0.28 & 2.31 & & 4.06 & 0.08 & 0.05 & 0.06 \\
\hline BER & 7.90 & 0.10 & 8.00 & 1.60 & 0.3 & 1.98 & & 3.97 & 0.05 & 0.04 & \\
\hline BOA & 7.80 & 0.2 & 8.00 & 1.64 & 0.35 & 1.91 & & 3.90 & 0.04 & 0.09 & 0.21 \\
\hline CAS & 7.98 & 0.02 & 8.00 & 1.88 & 0.15 & 1.79 & & 3.82 & 0.06 & 0.08 & 0.12 \\
\hline E 10 & 7.95 & 0.05 & 8.00 & 0.92 & •.14 & 3.34 & & 4.40 & 0.03 & 0.04 & \\
\hline Ell & 7.92 & 0.08 & 8.00 & 1.14 & 0.18 & 2.92 & & 4.24 & 0.04 & 0.04 & 0.06 \\
\hline LIB & 8.00 & & 8.00 & 1.61 & 0.16 & 2.27 & $\bullet .01$ & 4.05 & 0.02 & & 0.05 \\
\hline LIL & 8.00 & & 8.00 & 1.89 & 0.02 & 1.95 & & 3.86 & 0.01 & & 0.07 \\
\hline MET & 8.00 & & 8.00 & 1.75 & 0.05 & 2.16 & & 3.96 & 0.08 & 0.01 & \\
\hline NIJ & 8.00 & & 8.00 & 1.62 & 0.18 & 2.10 & & 3.90 & 0.01 & & 0.19 \\
\hline OKE & 7.92 & 0.08 & 8.00 & 1.99 & 0.03 & 1.88 & & 3.90 & 0.02 & 0.02 & 0.14 \\
\hline PAL & 7.93 & 0.07 & 8.00 & 1.89 & 0.02 & 2.05 & & 3.96 & 0.04 & 0.02 & 0.10 \\
\hline PIC & 7.96 & 0.04 & 8.00 & 1.28 & 0.47 & 2.24 & 0.01 & 4.00 & 0.02 & 0.01 & •.16 \\
\hline SEG & 7.90 & 0.10 & 8.00 & 1.97 & 0.06 & 1.85 & & 3.88 & 0.05 & 0.01 & 0.16 \\
\hline TOR & 7.88 & 0.12 & 8.00 & 1.43 & 0.38 & 2.28 & & 4.09 & 0.01 & 0.02 & \\
\hline TRA & 8.00 & & 8.00 & 1.20 & ๑. 15 & 2.83 & & 4.19 & 0.05 & 0.02 & \\
\hline $\mathrm{Y}$ & 7.93 & 0.07 & 8.00 & 1.51 & 0.34 & 2.10 & 0.01 & 3.96 & 0.02 & 0.06 & 0.08 \\
\hline Y $1 \mathrm{~A}$ & 7.99 & 0.01 & 8.00 & 1.52 & ๑. 16 & 2.38 & & 4.06 & 0.03 & 0.01 & 0.02 \\
\hline Y3 & 8.00 & & 8.00 & 1.54 & 0.13 & 2.35 & 0.01 & 4.03 & 0.01 & 0.04 & 0.04 \\
\hline Y7 & 7.96 & 0.04 & 8.00 & 1.62 & 0.17 & 2.21 & & 4.00 & 0.03 & 0.01 & 0.07 \\
\hline Y8 & 7.91 & 0.09 & 8.00 & 1.68 & 0.2 & 2.16 & 0.01 & 4.05 & 0.01 & 0.07 & 0.01 \\
\hline YUC & 8.00 & & 8.00 & 1.47 & .23 & 2.34 & & 4.04 & 0.04 & & 0.01 \\
\hline MAX & 8.00 & 0.2 & & 1.99 & 0.47 & 3.34 & 0.01 & 4.40 & 0.08 & 0.09 & 0.21 \\
\hline MIN & 7.86 & 0.00 & & 0.92 & 0.02 & 1.79 & 0.00 & 3.82 & 0.01 & 0.00 & 0.00 \\
\hline MEAN & 7.95 & 0.06 & & 1.57 & 0.19 & 2.24 & 0.00 & 4.01 & 0.03 & 0.03 & 0.07 \\
\hline
\end{tabular}




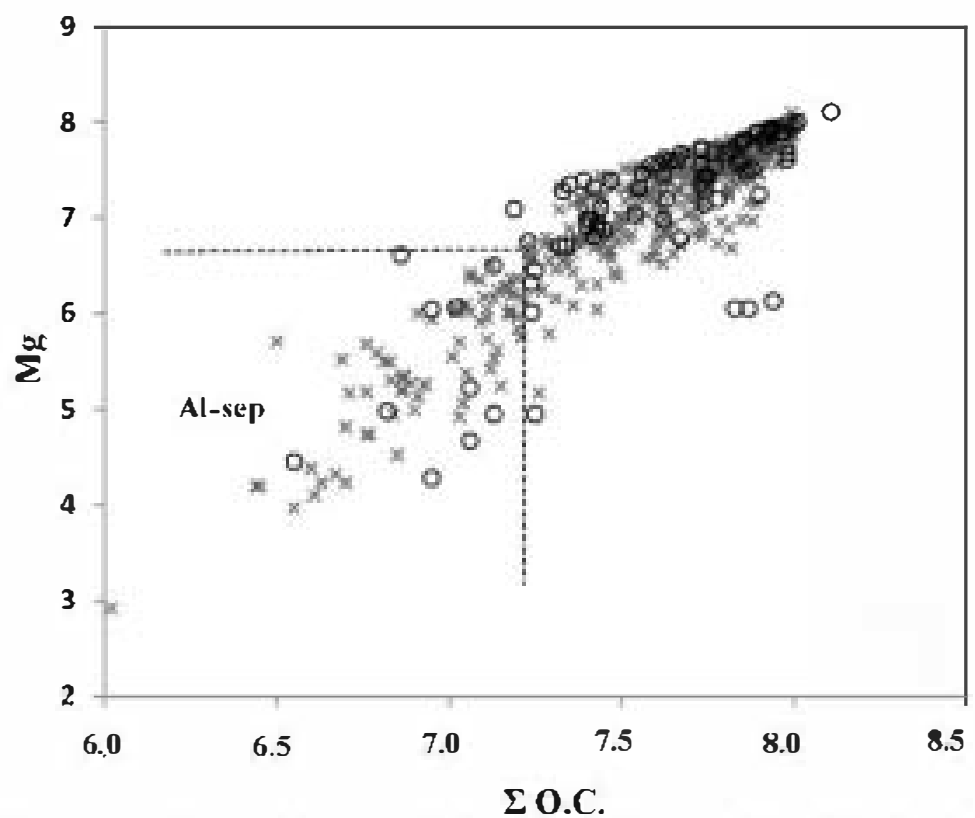

Figure 2. Mg content (p.h.u.c.) vs. total octahedral content, for a ta from the literature (O) and for data from the present study (x). The $t w \bullet$ kinds $\bullet$ a ta are projected in the same region of the plot.

Greek samples studied by Gienis et al. (2007). According to this classification mest palygerskites studied here correspond to Type II and magnesic palygorskites (Type III) are E10, E11, and TRA. Among these, the smallest $\mathrm{Al}$ content corresponds to E1 (192), the most rioctahedral palygerskite found in the present study.

All swuctural formulae calculated frem AEM analysis -f palygerskite studied here and in the literature are projected taking inte account the number of $\mathrm{M}$ (p.h.u.c.) vs. the octahedral content (total number of -ctahedral cations p.h.u.c.). When the samples studied here are compared to those in the literature, similar tendencies are found (Figure 3) and the data from the literature can als be classified int the types described previously.

With the literature data and new data coming from a very wide range of samples of different origins, however, a new type of palygorskite must be defined, namely the Type IV, 'aluminic palygerskite', the most

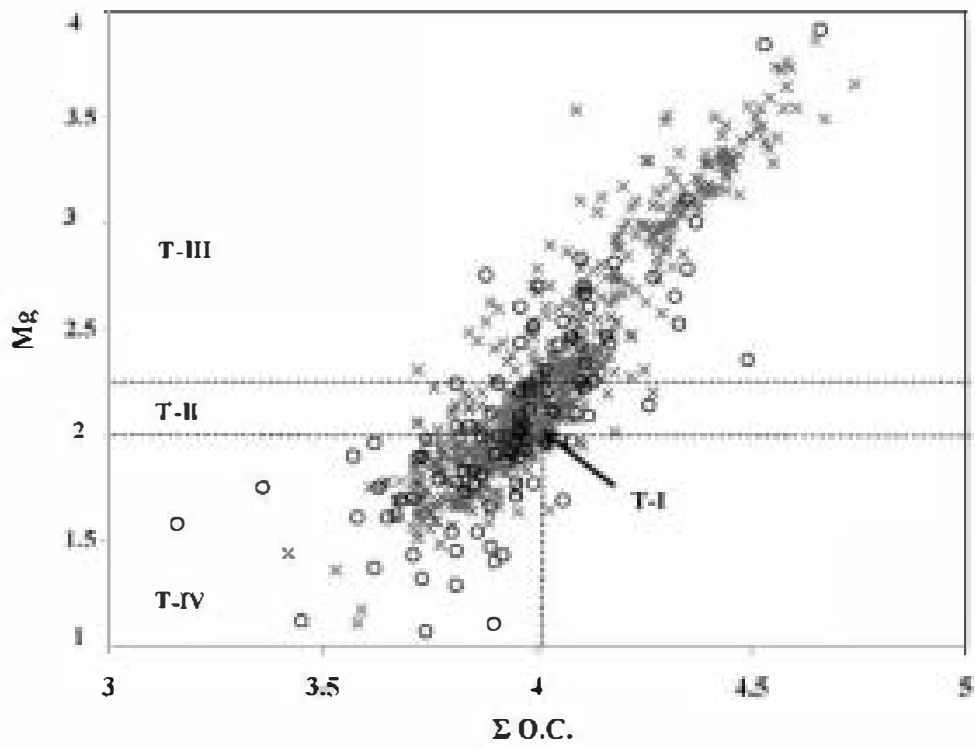

Figure 3. Number of Mg cations (p.h.u.c.) vs the total number of octahedral cations p.h.u.c. for palygorskites, for lata from the literature $(0)$ and for data from the presentstudy $(x)$. The diagram shews the four classifications (I-IV) described in the text. 
dioctahedral extreme, which contains palygorskites with a total number of octahedral cations (p.h.u.c.) of $<4$, with $R 3 / R 2>1$ and $M g<2$. Taking inte account the structural formulae proposed (Table 9) for the palygorskites from the present study, samples CAS, OKE, and SEG correspond to Type IV.

A category of Fe-rich palygorskite could be used when $\mathrm{Fe}>\mathrm{Al}$ in the different types described above. Palygorskite studied by Gionis et al. (2006) is an Fe-rich palygorskite, Type II; and Chryssikes et al. (2009) studied several palygorskites rich in Fe, that could be classified as Fe-rich, Types I, $\mathbf{1}$, and IIII. In the samples studied here, the Fe content can be large $(\leqslant .47)$ but none could be referred to as Fe-rich. This classification of palygorskites could be formulated with the variables proposed by Gionis et al. (2007): i.e. where $x, y$ refers to the palygorskite formula $y \mathrm{M}_{5} \mathrm{Si}_{8} \Theta_{2}(\bullet \mathrm{H})_{2} \cdot(1-y)$ $\left[x \mathrm{M}_{2} \mathrm{Fe}_{2}(1-x) \quad \mathrm{M}_{2} \mathrm{Al}_{2}\right] \mathrm{Si}_{8} \bullet_{2} \bullet(\bullet \mathrm{H})_{2}$. Taking int account that $y$ is an indication of the trioctahedral degree in palygorskite, $y$ is negative in Type $\mathrm{IV}, \sim$ in Type $I$, ranges between $\bullet .1$ and $\boldsymbol{\bullet . 2}$ in Type $\mathbf{M}$, and $>\mathbf{0 . 2}$ in Type MI (the most trioctahedral •ption). In the same formula, $x$ is an indication of the Fe content for all types -f Fe-rich palygorskites where $x \geqslant 0.5$.

A great variability in the structural formula of the twe minerals exists, especially in palygorskite. Several is morphic substitutions are possible and this means that the eccupancy of octahedral positions als varies. In both minerals, $\mathrm{Al}, \mathrm{Fe}$, and $\mathrm{Mg}$ can exist with very variable prepertions. Martin-Vivaldi and Cane-Ruiz (1956) said, "the minerals of the palygorskite-sepiolite group occupy the region of discontinuity between dioctahedral and triectahedral minerals," and comparing the samples in terms of octahedral occupancy is very useful. T• compare the tw॰ minerals from their structural data the relation between $\mathrm{Si} / \mathrm{Mg}$ cations and the occupancy of the octahedral position (in percentage) was used (Figure 4). The results corresponding to the structural formulae proposed for the samples studied in the present work are projected in the same graph as data taken from the literature. In the latter, greater dispersion was found due to the variety $\bullet$ methods of analyses and probably to the presence of impurities, but all samples are projected onto one curve. Three groups emerged: a group of samples of palygorskite which have the largest $\mathrm{Si} / \mathrm{M}$ rati and octahedral vacancies, a group of sepiolite samples with smaller values of these two variables, and a third group of samples in which both sepiolites and palygorskites are plotted. Mg-palygorskites and $\mathrm{Al}$-sepiolites are plotted between the ordinary palygorskite and sepielite groups.

The compositional limit between the twe minerals was found by studying the oxides content and taking inte account the fact that the samples studied here represent the general tendency found in the literature for these fibrous clay minerals (Figure 4).The mean $\mathrm{Si}_{2}$ content -f the sepiolites and palygorskites is similar, as can be seen when comparing the mean values of major oxides contents obtained for the samples studied with the theoretical formulae of both minerals. The $\mathrm{Si}_{2}$ content is slightly smaller in sepielite than in palygorskite and smaller than the ideal content for each mineral in seneral, as expected, taking inte account that in all

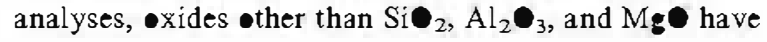

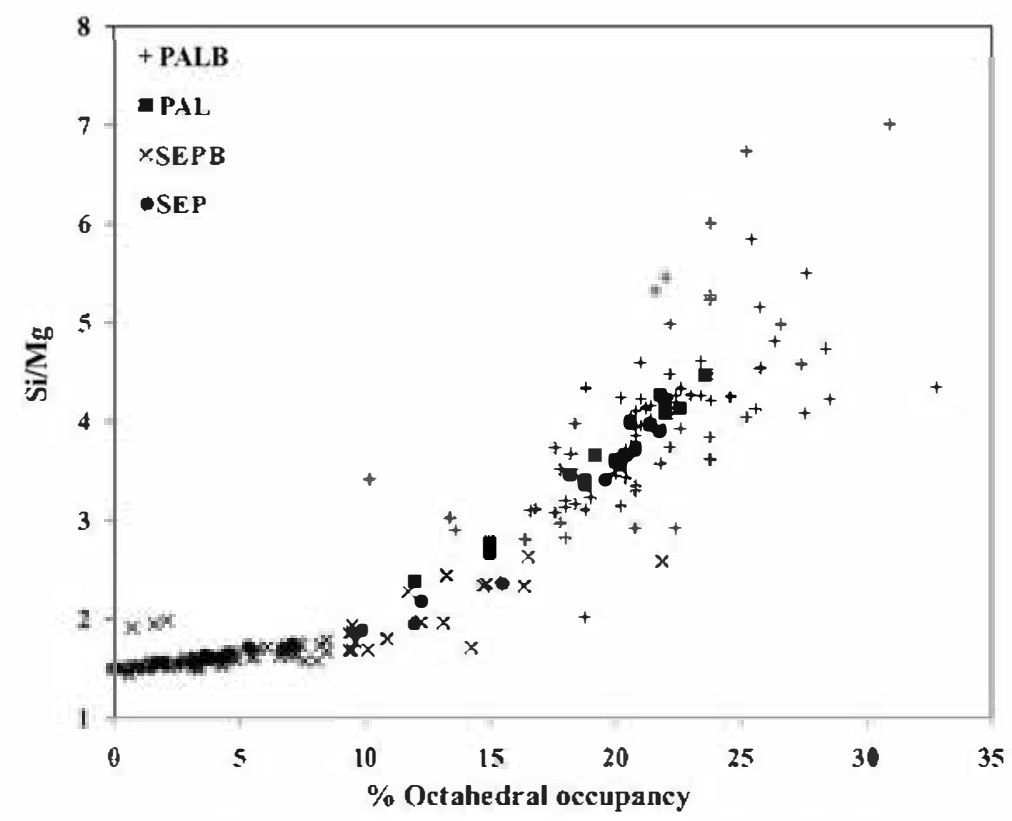

Figure 4. Comparisen between the sepielites and palygorskites from their structural formulae. The relation between Si/Mg and the -ctahedral occupancy $(\%)$ shows thesametendency both for ata from the literature and forthe data $\bullet$ btained in the present study and sepiolite and palygorskite are projected in a continuous region. 


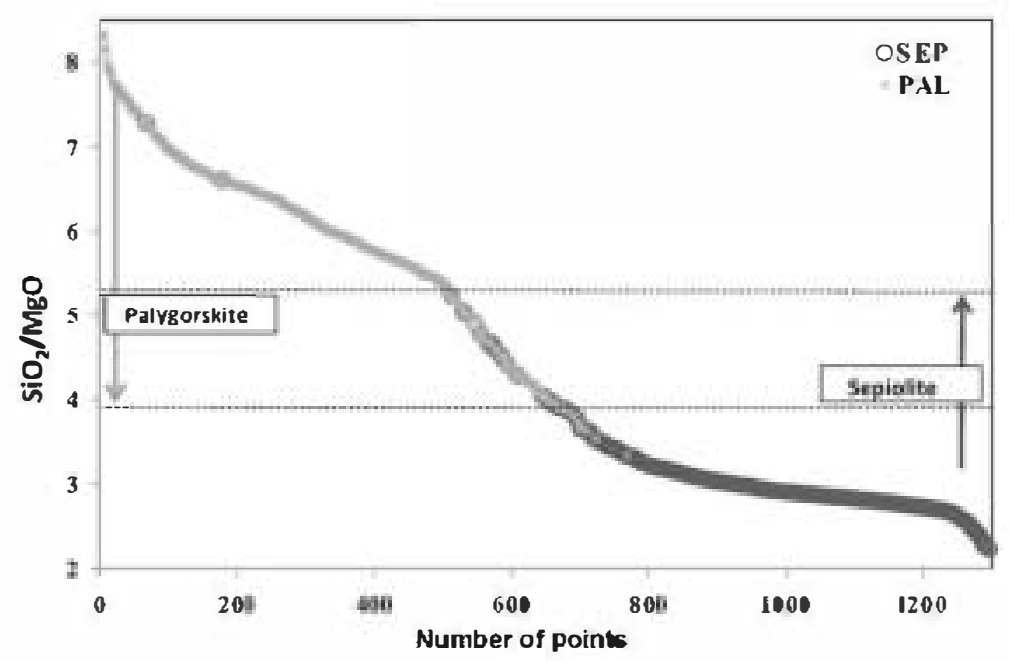

Figure 5. $\mathrm{SiO}_{2} / \mathrm{MgO}$ ratios for all AEM analyses, ordered from the largest to the smallest values.

been detected. Only three sepiolites (XIX, YUN, and LIE) and one palygorskite (LN) have a little more silica than these ideal compositions, indicating that these samples may contain adsorbed silica, especially sample XIX, in agreement with the structural formulae obtained.

The best way to compare the chemical composition is to compare the contents of the main oxides. When all of the particles analysed are plotted from the greatest to the smallest values of the relation between $\mathrm{Si}_{2}$ and $\mathrm{Mg} \bullet$, a continuous curve is •btained (Figure 5) and no break in the data between palygorskites and sepiolites was found. On the contrary, both minerals overlap and a continuous compositional variation exists from the ideal sepiolite to the most Al-rich palygorskite (points with the largest $\mathrm{Si}_{2} / \mathrm{Mg}$ values). Each type of mineral (sepiolite and palygorskite) is projected into different domains. Løgically, sepiolite plots in positions nearest to the smallest $\mathrm{Si}{ }_{2} / \mathrm{Mg}$ values. Palygorskite can have $\mathrm{Si}{ }_{2} /$ Mg values of between $~ 7.5$ and 3.0 and sepielite from $\sim 1.5$ to 4.5 , but in the range $3.4-4.5$ both minerals are possible.

The same kind of plot (Figure 6) and conclusions are -btained if the amounts of different $\bullet$ xides of octahedral cations are considered $\left(\mathrm{Al}_{2} \mathrm{O}_{3}+\mathrm{Fe}_{2} \boldsymbol{\omega}_{3} / \mathrm{Mg} \mathbf{0}\right)$. Again, each type of mineral (sepiolite and palygorskite) is prejected inte a different demain, but the tov demains -verlap. Sepiolite, logically, is projected inte the positions nearest to the smallest $\left.\left(\mathrm{Al}_{2} \boldsymbol{\omega}_{3}+\mathrm{Fe}_{2}\right)_{3}\right) / \mathrm{Mg}$ values. Palygorskite can have $\left(\mathrm{Al}_{2} \mathrm{O}_{3}+\mathrm{Fe}_{2} \mathrm{O}_{3}\right) / \mathrm{MgO}$ between $\sim .25$ and 2.2 and sepilite fr $\bullet$ t $\bullet \sim 1 . \bullet$, but in the range between $\bullet .25-1$ both minerals are possible.

Several samples, therefore, have intermediate chemical composition between the most extreme members,

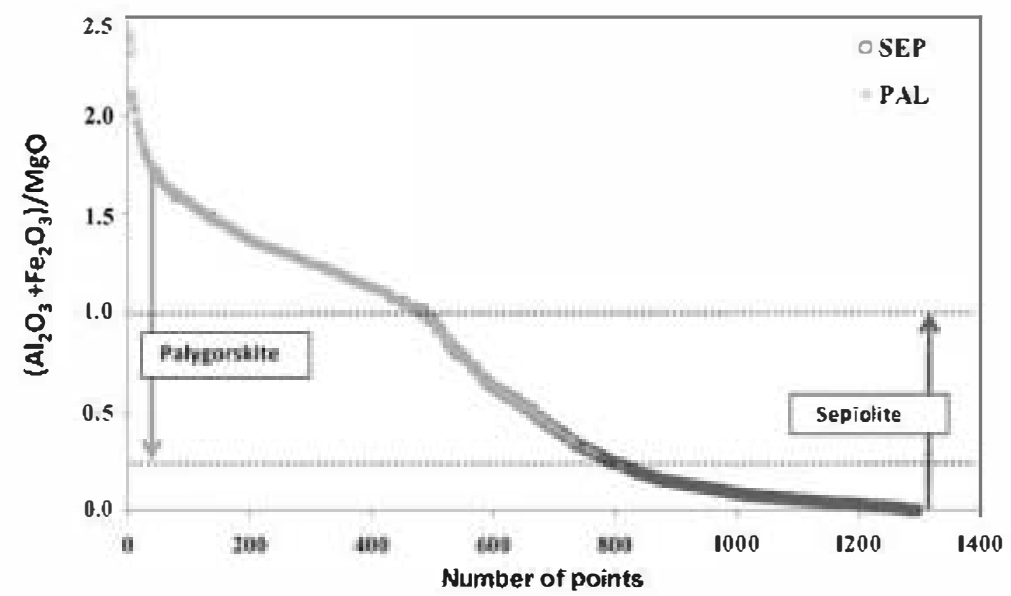

Figure 6. $\left(\mathrm{Al}_{2} \mathrm{O}_{3}+\mathrm{Fe}_{2} \mathrm{O}_{3}\right) / \mathrm{M} \mathrm{O}$ ratios for all the $\mathrm{AEM}$ analyses, ordered from the largest to the smallest values. 

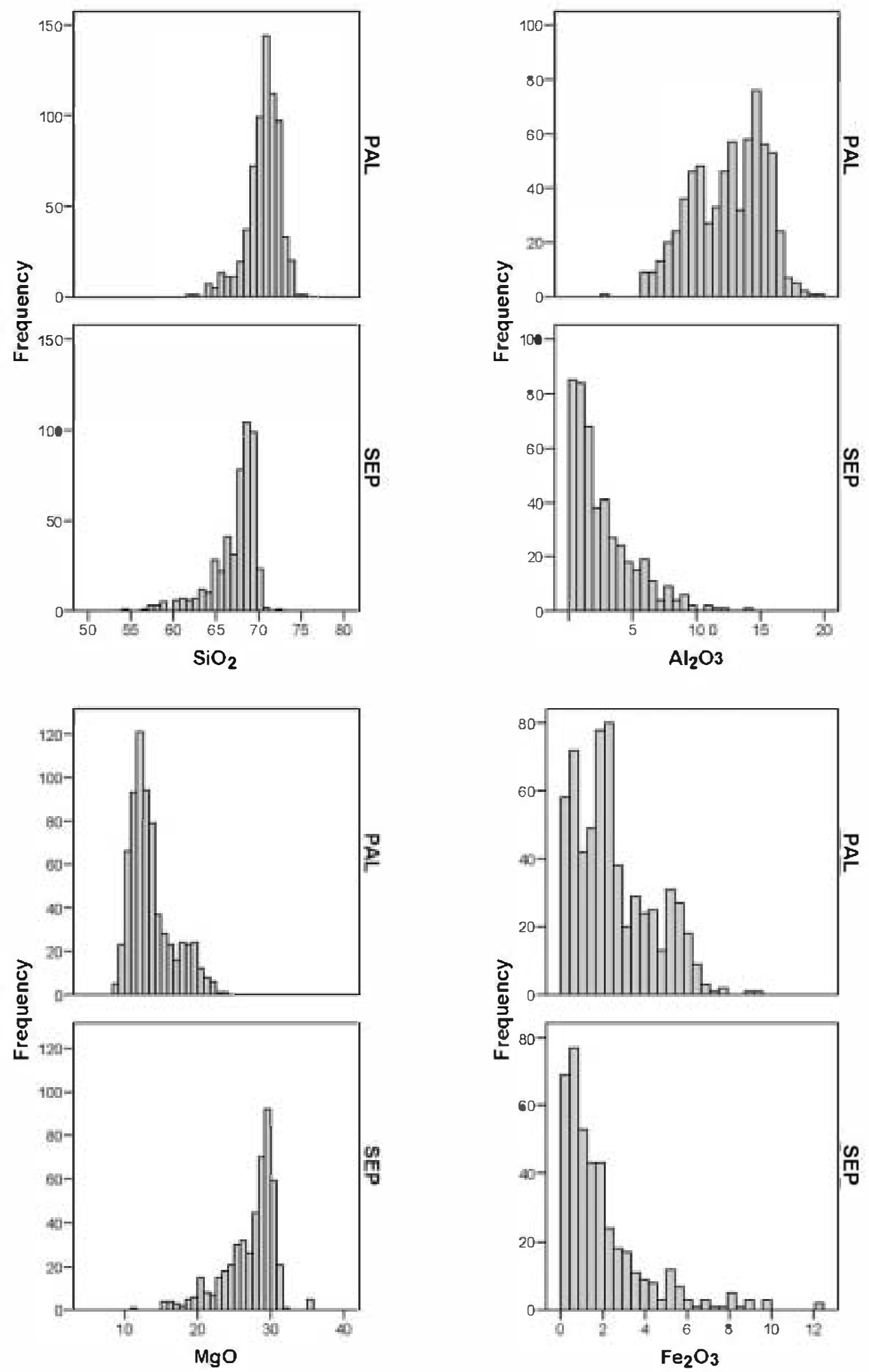

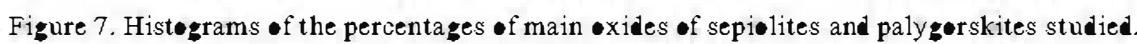


Table 10. Mean values of tetrahedral and octahedral cations, $\mathrm{Si} / \mathrm{Mg}$, and $\mathbf{R} 3 / \mathbf{R} 2$ for $\mathrm{Al}$-sepiolite samples on the basis of $\mathbf{O}_{2}(\mathbf{O H})_{2}$ (as palygorskite) and for Mg-palygorskite on the basis of $\mathbf{O}_{3}(\mathbf{O H})_{4}$ (as sepiolite).

\begin{tabular}{|c|c|c|c|c|c|c|c|c|c|c|c|c|c|}
\hline & $\mathrm{Si}$ & ${ }^{\mathrm{rV}} \mathrm{Al}$ & $\Sigma \tau$ & ${ }^{\mathrm{VI}} \mathrm{Al}$ & $\mathrm{Fe}^{3+}$ & $\mathrm{Mg}$ & $\mathrm{Ti}$ & $\Sigma_{0}$ & $\mathrm{Ca}$ & $\mathrm{K}$ & $\mathrm{Na}$ & $\mathrm{Si} / \mathrm{Mg}$ & $\mathbf{R} 3 / \mathbf{R} 2$ \\
\hline E10 & 12.12 & 0.03 & 12.15 & 1.45 & 0.21 & 5.15 & & 6.81 & 0.06 & 0.06 & 0.00 & 2.35 & 0.32 \\
\hline Ell & 12.09 & 0.06 & 12.15 & 1.82 & 0.26 & 4.48 & & 6.56 & 0.09 & 0.06 & 0.08 & 2.70 & 0.46 \\
\hline TRA & 12.23 & & 12.23 & 1.86 & $\bullet .24$ & 4.30 & & 6.40 & 0.07 & 0.03 & & 2.85 & 0.49 \\
\hline BAT & 7.87 & 0.18 & 8.05 & 0.36 & 0.13 & 4.05 & 0.05 & 4.59 & 0.07 & 0.05 & 0.02 & 1.94 & •. 12 \\
\hline HUN & 7.66 & 0.34 & 8.1 & 0.39 & 0.23 & 4.01 & & 4.62 & 0.90 & 0.15 & 0.17 & 1.91 & 0.15 \\
\hline LIE & 7.90 & 0.10 & 8.00 & 0.71 & 0.21 & 3.59 & & 4.51 & 0.05 & 0.01 & 0.01 & 2.20 & 0.26 \\
\hline POL & 7.57 & 0.42 & 7.99 & 0.78 & 0.43 & 3.06 & 0.10 & 4.37 & 0.14 & 0.11 & & 2.47 & 0.40 \\
\hline
\end{tabular}

e.g. samples POL sepiølite and E10 palygerskite (Tables 4 , 5) have similar chemical compesitions and as a censequence are prejected in the same regien of the plet. If histegrams correspending to the main exides contents (Figure 7) are considered, in all cases both sepiolite and palygerskite fields everlap and sepielite and palygerskite can cøntain similar amøunts $\bullet$ the four main $\bullet$ xides. Ne chemical differences exist within a certain number $\bullet$ analyses of sepielites and palygerskites which have a compesition that might be referred to as intermediate. These samples are Mg-palygorskites and Al-sepielites, the compesitions of which are similar that if the structural formula were fitted as if they were the 'ther' mineral (Table 10), fitting sepielite as palygerskite (with 21 negative charges) and palygerskite as sepielite (with 32 negative charges), acceptable results frem the p•int -f view of eccupancy of pesitiens and balance of charges can be reached.

Although slight differences have been found, the analyses indicate a very narrew cempesitional range in each sample, as illustrated by their small standard deviation. Nevertheless, the samples display a large compesitional range from each other. This indicates that each sample has its own features, which may be as a

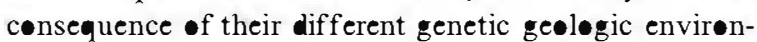
ments. Data from the collection studied here are consistent with these published for these minerals, even taking int accunt that the literature data can be affected by the presence of impurities. Theref $\bullet$ re, a continueus range of chemical compesition exists (Figures 1-7) and a compesitienal gap is absent. On the contrary, chemical compesitiens can be se similar that a certain degree of pelymerphism between Alsepielite and Mg-palygerskite exists.

The results $\bullet$ btained raise tw questions:

(1) What kinds of conditions are necessary to precipitate sepielite or palygerskite frem a sølution with the same compesition (with respect to the main -xides)?

(2) What structural arrangements de Al-sepielite and Mg-palygerskite have? Are the excess $\mathrm{Mg}$ and $\mathrm{Al}$ within specific demains in the structures or hømegene»usly distributed aleng the ribbens?

\section{CONCLUSIONS}

Both sepielite and palygerskite can have a certain degree of tetrahedral substitution ( $<2 \%$ generally).

Theoretical sepielite has eight ectahedral pesitiens, all of which are filled by $\mathrm{Mg}$. S॰me ectahedral substitutions of $\mathrm{Mg}$ for $\mathrm{Al}$ and/or Fe are pessible which induce an increase in the number of ectahedral

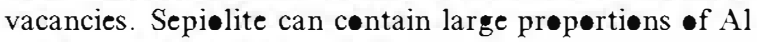
and be considered Al-sepielite.

Palygerskite has, as is well kn॰wn, a greater likelihoed of ectahedral substitution than sepielite. Alpalygerskite, cemmen palygerskite, Mg-palygerskite, and eccasienally Fe-palygerskite als exist

Accoring to the data presented here, ne compesitional gap exists between sepielite and palygerskite.

Al-rich sepielites and the Mg-rich palygerskites can have similar cempesitiens: $\mathrm{Si} / \mathrm{Mg}$ between 3.1 and 4.5 and $\left(\mathrm{Al}_{2} \mathrm{O}_{3}+\mathrm{Fe}_{2} \mathrm{O}_{3}\right) \mathrm{Mg} \bigcirc$ between 0.25 and 1 .

\section{ACKNOWLEDGMENTS}

The authors are grateful to Catherine Doyle for checking and improving the English. They are also very grateful to Drs Selahattin Kadir and Yuanfeng Cai whose constructive commets improved the manuscript. Financial support from the Spanish CICYT (project CGL200609843) and 'Servicio de Investigación de la U.C.M. (Grupo 9103 B6)' are also acknowledged.

\section{REFERENCES}

Akbulut, A. and Kadir, S. (2003) The geology and origin of sepiolite, palygorskite and saponite in Neogene lacustrine sediments of the Serinhisar-Acipayam Basin, Denizli, SW Turkey. Clays and Clay Minerals, 51, 279-292.

Arauzo Perez, A., González López, J.M., and López Aguayo, F. (1989) Primeros datos sobre la mineralogía y génesis del yacimiento de sepiolita de Mara (prov. de Zaragoza). Boletín de la Sociedad Española de Mineralogía, 12, $329-340$.

Artioli, G. and Galli, E. (1994) The crystal structures of orthorhombic and monoclinic palygorskite. Materials Science F•rum, 166-169, 647-652.

Artioli, G., Galli, E., Burattini, E., Cappuccio, G., and Simeoni, S. (1994) Palygorskite from Bolca, Italy: a characterization by high-resolution synchrotron radiation powder diffraction and computer modelling. Neues Jahrbuch fur Mineral ogie Mønatshefte, 5, 217-229. 
Bradley, W.F. (1940) The structural scheme of attapulgite. American Mineral•gist, 25, 405-411.

Brauner, K. and Presinger, A. (1956) Struktur und Entstehung des Sepioliths. Tschermak's Mineraløgische und Petr@graphische Mitteilungen, 6, 1-2, pp. 120-140.

Chahi, A., Fritz, B., Duplay, J., Weber, F., and Lucas, J. (1997) Textural transition and genetic relationship between precursor stevensite and sepiolite in lacustrine sediments (Jbel Rhassoul, Morocco). Clays and Clay Minerals, 45, $378-389$

Chahi, A., Petit, S., and Decarreau, A. (2002) Infrared evidence of dioctahedral-trioctahedral site occupancy in palygorskite. Clays and Clay Minerals, 50, 306-313.

Chen, T., Wang, H., Zhang, X., and Zheng, N. (2008) SAED and HRTEM investigation of palygorskite. Act Gel•gic Sinica, 82, 385-391.

Chryssikos, G.D., Gionis, V., Kacandes, G.H., Stathopoulou, E.T., Suárez, M., García-Romero, E., and Sánchez del Rio, M. (2009) Octahedral cation analysis of palygorskite by near-infrared spectroscopy. American Mineraløist, 94, $200-203$.

Corma, A., Mifsud, A., and Sanz, E. (1987) Influence of the chemical composition and textural characteristics of palygorskite on the acid leaching of octahedral cations. Clay Minerals, 22, 225-232.

Drits, V.A. and Sokolova, G.V. (1971) Stucture of palygorskite. S•viet Physics Crystalløgraphy, 16, 288-231.

Ece, Ö.I. (1998) Diagenetic transformation of magnesite pebbles and cobbles to sepiolite (meerschaum) in the Miocene Eskişehir lacustrine basin, Turkey. Clays and Clay Minerals, 46, 436-445.

Fahey, J.J., Ross, M., and Axerod, J.M. (1960) Loughlinite, a new hydrous sodium magnesium silicate. American Mineral•gist, 45, 27-281.

Galán, E. and Carretero, I. (1999) A new approach to compositional limits for sepiolite and palygorskite. Clays and Clay Minerals, 47, 399-409.

Galán, E., Brell, J.M., La Iglesia, A., and Robertson, R.H.S. (1975) The Caceres palygorskite deposit, Spain. Proceedings of the International Clay Conference. Applied Publishing Ltd. Willmette, Illinois 60091, USA, pp. 81-94.

Garcia-Romero, E., Suárez, M., and Bustillo, A. (2004) Characteristics of a $\mathrm{Mg}$-palygorskite in Miocene rocks, Madrid Basin (Spain). Clays and Clay Minerals, 52, 484-494.

García-Romero, E., Suárez, M., Oyarzun, R., López-García, J.A., and Regueiro, M. (2006) Fault-hosted palygorskite from the Serrata de Ni jar Deforation Zone (SE Spain). Clays and Clay Minerals, 54, 324-332.

García-Romero, E., Suárez, M., Santaren, J., and Alvarez, A. (2007) Crystallochemical characterization of the palygorskite and sepiolite from the Allou Kagne deposit, Senegal. Clays and Clay Minerals, 6, 606-617.

Gionis, V., Kacandes, G.H., Kastritis, I.D., and Chryssikos, G.D. (2006) On the structure of palygorskite by mid- and near-infrared spectroscopy. American Mineraløgist, 91, $1125-1133$.

Gionis, V., Kacandes, G.H., Kastritis, I.D., and Chryssikos, G.D. (2007) Combined near-infrared and X-ray diffraction investigation of the octahedral sheet composition of palygorskite. Clays and Clay Minerals, 55, 543-553.

Giusteto, R., Levy, D., and Chiari, G. (2006) Crystal structure refinement of Maya Blue pigment prepared with deuterated indigo, using neutron powder diffraction. Eurøpen Joumal - Mineral $\bullet y, 18,629-640$

Güven, N. (1992) The coordination of aluminum ions in the palygorskite structure. Clays and Clay Minerals, 40, $457-461$

Imai, N. and Otsuka, R. (1984) Sepiolite and palygorskite in
Japan. Pp. 211-232 in: Palygorskite-Sepiølite, currences, Genesis and Uses (A. Singer and E. Galán, editors). Developments in Sedimentology, 37, Elsevier, Amsterdam. Jamoussi, F., Ben Aboud, A., and López-Galindo, A. (2003) Palygorskite genesis through silicate transformation in Tunisian continental Eocene deposits. Clay Minerals, 38, $187-199$

Jones, B.F. and Galán, E. (1991) Sepiolite and palygorskite. Pp. 631-674 in: Hydrous Phyllosilicates (Exclusive of Mics) (S.W. Bailey, editor). Reviews in Mineralogy, 19. Mineralogical Society of America, Washington, D.C.

Kadir, S., Bas, H., and Karakas, Z. (2002) Origin of sepiolite and loughlinite in a Neogene volcano-sedimetary lacustrine environment, Mihaliç̧̧ik-Eskişehir, Turkey. The Canadian Mineraløgist, 40, 1091-1102.

Krekeler, M.P.S. and Guggenheim, S. (2008) Defects in microstructure in palygorskite-sepiolite minerals: A transmission electron microscopy (TEM) study. Applied Clay Science, 39, 98-105

Li, Z., He, K., Yin, L., Xiong, F., and Zeng, Y.C. (2007) Crystallochemistry of Fe-rich palygorskite from eastern China. Clay Minerals, 42, 453-461.

López-Galindo, A. (1987) Paligorskita en materiales cretácicos de la zona subbetica. Origen. Boletín de la Sociedad Española de Mineraløgía, 10-2, 131-139.

López-Galindo, A. and Sánchez Navas, A. (1989) Criterios morfológicos, cristalográficos y geooquímicos de diferenciación entre sepiolitas de origen sedimentario e hidrotermal. Boletín de la Sociedad Española de Mineralıgía, 12, $375-383$

López-Galindo, A., Aboud, B., Fenoll Hach-Ali, P., and Casas Ruiz, J. (1996) Mineralogical and geochemical characterization of palygorskite from Gabasa (NE Spain). Evidence of a detrital precursor. Clay Minerals, 31, 33-44.

López-Galindo, A., Fenoll Hach-Alí, P., Pushkarev, A.V., Lytovchenko, A.S., Baker, J.H., and Puskarova, R.A. (2008) Tritium redistribution between water and clay minerals. Applied Clay Science, 9, 151-159.

Lorimer, G.W. and Cliff, G. (1976) Analytical electron microscopy of minerals. Pp. 506-519 in: Electron Micrøscopy in Mineraløgy (H.R. Wenk, editor). SpringerVerlag. Berlin.

Magalhaes, C.A., Da Luz, A.B., Baltar, L.M., De Oliveira, C.H., and Becerra, F.J. (2008) Influence of morphology and surface charge on the suitability of palygorskite as drill fluid. Applied Clay Science, 42, 597-600.

Martín-Vivaldi, J.L. and Cano-Ruíz, J. (1956) Contribution to the study of sepiolite: II. Some considerations regarding the mineralogical formula. Proceedings of four National Conference on Clays and Clay Minerals. National Academy of Sciences, National Research Council, 156, $173-176$

Martín-Vivaldi, J.L. and Fenoll, P. (1970) Palygorskites and sepiolites (Hormites). Pp. 553-573 in: Differential Thermal Analysis (R.C. Mackenzie, editor). Academic Press, London.

Mumpton, F.A. and Roy, R. (1958) New data on sepiolite and attapulgite. National Academy of Sciences - Nati@nal Research Council, pp. 136-143.

Neaman, A. and Singer, A. (2000) Kinetics of palygorskite hydrolysis in dilute salt solutions. Clay Minerals, 35, 433-441.

Newman, A.C.D. and Brown, G. (1987) The chemical constitution of clays. Pp. $1-129$ in: Chemistry of Clays and Clay Minerals (A.C.D. Newman, editor). Monograph 6, Mineralogical Society, London.

Paquet, H., Duplay, J., Valleron-Blanc, M.M., and Millot, G. (1987) Octahedral compositions of individual particles in smectite-palygorskite and smectite-sepiolite assemblages. Proccedings of the International Clay Conference. Denver, 
1985 (L.G. Schultz, H. Van Olphen, and F.A. Mumpton, editors). The Clay Minerals Society, Bloomington, Indiana, pp. 73-77

Post, J.L. and Crawford, S. (2007) Varied forms of palygorskite and sepiolite from different geologic systems. Applied Clay Science, 26, 232-244.

Post, J.E. and Heaney, P. J. (2008) Synchrotron powder X-ray diffraction study of the structure and dehydration behavior of palygorskite. American Mineral gist, 93, 667-675.

Rogers, L.E., Quirk, J., and Norrish, K. (1956) Occurrence of an aluminium-sepiolite in a soil having unusual water relationships. Joumal of Soil Science, 7, 177-184.

Santaren, J., Sanz, J., and Ruitz-Hitzky, E. (1990) Structural fluorine in sepiolite. Clays and Clay Minerals, 38, 63-68.

Serna, C., Rautureau, M., Prost, R., Tchoubar, C., and Serratosa, J.M. (1977) Etude de la sépiolite a l'aide des domnées d ela micrsocopie électroniqe, de l'analyse thermopondérale et de la spectroscopie infrarouge. Bulletin Groupe Française de Argiles, XXVI, 153-163.

Siddiki, M.H.K. (1984) Occurrence of palygorskite in the Deccan Trap formation in India. Pp. 243-250 in: Palygorskite-Sepiølite, currences, Genesis and Uses (A. Singer and E. Galán, editors). Developments in Sedimentology, 37, Elsevier, Amsterdam.

Singer, A. and Norrish, K. (1974) Pedogenic palygorskite occurrences in Australia. American Mineralogist, 59, $508-517$

Singer, A., Stahr, K., and Zarei, M. (1998) Characteristics and origin of sepiolite (Meerschaum) from Central Somalia. Clay Minerals, 33, 349-362.

Springer, G. (1976) Falcondoite, a nickel analogue of sepiolite. The Canadian Mineral•gist, 14, 407-408.

Suárez, M. and García-Romero, E. (2006) Macroscopic palygorskite form Lisbom Volcanic Complex. Eur pen Journal of Mineraløgy, 18, 119-126.

Suárez, M., Robert, M., Elsass, F., and Martín Pozas, J.M. (1994) Evidence of a precursor in the neoformation of palygorskite - new data by analytical electron microscopy. Clay Minerals, 29, 255-264.

Suárez, M., García-Romero, E., and Sánchez del Río, M.
(2006) Aproximación a la composición química de la palygorskita a partir de los datos de difracción de rayos-x. Macl 6, 667-470.

Suárez, M., García-Romero, E., Sánchez del Río, M., Martinetto, P., and Dooryhée, E. (2007) The effect of the octahedral cations on the dimensions of the palygorskite cell. Clay Minerals, 42, 287-297.

Taulet, E., Proenza, J.A., Galí, S., Lewis, J.F., Labrador, M., García-Romero, E., Suárez, M., Longo, F., and Bloise, G. (2009) Ni-sepiolite falcondoite in garnierite mineralization from Falcondo Ni-laterite deposit, Dominica Republic. Clay Minerals, 44, 431-450.

Tien, P.L. (1973) Palygorskite from Warren Quarry, Enderby, Leicestershire, England. Clay Minerals, 10, 27-34.

Torres Ruíz, J., López- Galindo, A., González-López, J.M., and Delgado, A. (1994) Geochemistry of Spanish sepiolitepalygorskite deposits: Genetic considerations based on trace elements and isotopes. Chemical Geløgy, 112, 221-245.

Verrecchia, E.P. and Le Coustumer, M.N. (1996) Occurrence and genesis of palygorskite and associa ted clay minerals in a Pleistocene calcrete Complex, Sde Boqer, Negev Desert, Israel. Clay Minerals, 31, 183-202.

Weaver, E. (1984) Origin and geologic implications of the palygorskite deposits of S.E. United States. Pp. 39-58 in: Palygørskite-Sepiølite, currences, Genesis and Uses (A. Singer and E. Galán, editors). Developments in Sedimentology, 37, Elsevier, Amsterdam.

Weaver, C.E. and Polland, L.D. (1973) The Chemistry f Clay Minerals. Developments in Sedimentology, Elsevier, Amsterdam, $213 \mathrm{pp}$.

Yalçin, H. and Bozkaya, Ö. (2004) Ultramafic rock hosted vein sepiolite occurrences in the Ankara ophiolitic mélange, Central Anatolia, Turkey. Clays and Clay Minerals, 52, 227-239.

Zaaboub, N., Abdel jaouad, S., and López-Galíndo, A. (2005) Origin of fibrous clays in Tunisian Paleogene continental deposits. Joumal of African Earth Sciences, 43, 491-504. 